\title{
Symmetries, Dynamics, and Control for the 2D Kolmogorov Flow
}

\author{
Nejib Smaoui (iD) \\ Department of Mathematics, Kuwait University, P.O. Box 5969, 13060 Safat, Kuwait \\ Correspondence should be addressed to Nejib Smaoui; nsmaoui64@yahoo.com
}

Received 13 July 2017; Accepted 19 March 2018; Published 13 May 2018

Academic Editor: Sigurdur F. Hafstein

Copyright (C) 2018 Nejib Smaoui. This is an open access article distributed under the Creative Commons Attribution License, which permits unrestricted use, distribution, and reproduction in any medium, provided the original work is properly cited.

\begin{abstract}
The symmetries, dynamics, and control problem of the two-dimensional (2D) Kolmogorov flow are addressed. The 2D Kolmogorov flow is known as the 2D Navier-Stokes (N-S) equations with periodic boundary conditions and with a sinusoidal external force along the $x$-direction. First, using the Fourier Galerkin method on the original 2D Navier-Stokes equations, we obtain a seventh-order system of nonlinear ordinary differential equations (ODEs) which approximates the behavior of the Kolmogorov flow. The dynamics and symmetries of the reduced seventh-order ODE system are analyzed through computer simulations for the Reynolds number range $0<R_{e}<26.41$. Extensive numerical simulations show that the obtained system is able to display the different behaviors of the Kolmogorov flow. Then, we design Lyapunov based controllers to control the dynamics of the system of ODEs to different attractors (e.g., a fixed point, a periodic orbit, or a chaotic attractor). Finally, numerical simulations are undertaken to validate the theoretical developments.
\end{abstract}

\section{Introduction}

In recent years, a lot of efforts have been devoted to construct dynamical systems that arise from solving the 2D NavierStokes equations. In the literature, the dynamics of the Navier-Stokes equations were approximated by using several reduced order models [1-20]. The concept of approximate inertial manifold (AIM) (see [1-11] and references therein) and Fourier Galerkin methods [12-20] is among the methods used to obtain such reduced order systems with the task of approximating the long-time behavior of the 2D NavierStokes equations. The behavior of the 2D Navier-Stokes equations depends on the nature of the forcing. When the force is of a single mode, the 2D N-S equations with periodic boundary conditions are known as the 2D Kolmogorov flow.

During the last three decades, numerous numerical studies of the 2D Kolmogorov flow with different forcing terms have appeared [11-29]. In 1981, Franceschini et al. [14-17] constructed a system of ODEs approximating the dynamics of the Kolmogorov flow when the force acts on the mode $(2,-1)$. In [15], a number of steady states and Hopf bifurcation have been observed up to Reynolds number 100. In 1996,
Armbruster et al. [25] analyzed the dynamics of the 2D Navier-Stokes equation when the force acts on the mode $(0,2)$ by exploiting as much as possible the symmetries in the problem and by using the Karhunen-Loeve decomposition (K-L). Later on and in 1997, Smaoui and Armbruster [27] described a computationally effective way to obtain a reduced order equivariant system of the $2 \mathrm{D} \mathrm{N}-\mathrm{S}$ equations using K-L decomposition with symmetries; the reduced order system obtained consists of 12 nonlinear ODEs. They showed that when $\mathrm{Re}=16.6$, the ODE system exhibits the same dynamics as the original simulation of the $2 \mathrm{D} \mathrm{N}-\mathrm{S}$ equations. However, the obtained Galerkin system does not show signs of a homoclinic behavior as observed in the original PDE simulation [25].

On the other hand, the control problem of the NavierStokes equations and especially to the 2D Navier-Stokes equations has not been completely investigated (see [3038] and the references therein). In 2003, using a global pinning coupling strategy, Guan et al. [34] designed an adaptive controller to control flow turbulence governed by the 2D Navier-Stokes equations. In 2009, Gambino et al. [35] designed an adaptive controller to drive the state of the system 
to the stationary solution. Recently, Smaoui and Zribi [36-38] constructed reduced order ODE models using the truncated Fourier expansion method for approximating the dynamics of the $2 \mathrm{D} \mathrm{N}$-S equations when the force acts on the mode $(0, \alpha)$. They showed that, for $\alpha=4$, the dynamics of the reduced order models exhibit periodic doubling bifurcation leading to chaotic attractors. In addition, they designed Lyapunov based control laws to drive the states of the reduced order model to the basic state solution and to synchronize two reduced order ODE models having different Reynolds and starting from two different initial conditions.

In this paper, we construct a system of seven ODEs that approximates the dynamics of Kolmogorov flow when the force acts on the mode $(0,2)$. It should be noted that the approach used in this paper to construct the system of ODEs is different than the one used in [36-38]. In addition, unlike the system obtained earlier by Smaoui and Armbruster [27], this system shows a homoclinic gluing bifurcation similar to the one observed in the original PDE simulations [25]. Furthermore, Lyapunov based control laws are designed to control the dynamics of the system for a given Re.

The paper is organized as follows. In Section 2, the 2D Navier-Stokes equations are presented and a seventh-order nonlinear ODE system is obtained to approximate the behavior of these equations. The reduced order ODE system is also analyzed in Section 2. Section 3 presents the design of a control law which is used to regulate the states of the reduced order ODE system to a desired fixed point. Section 4 deals with the design of a control scheme to synchronize two reduced order systems obtained from the $2 \mathrm{D} \mathrm{N}-\mathrm{S}$ equations having the same or different Reynolds number but they start from different initial conditions. Finally, some concluding remarks are given in Section 5.

\section{The 2D Kolmogorov Flow}

2.1. The Seven-Mode Reduced Order System of the 2D Kolmogorov Flow. The "basic 2D Kolmogorov flow" $\vec{u}=$ ( $\alpha \sin \alpha y, 0)$ was introduced by Kolmogorov in 1958 as an example on which to study transition to turbulence [39]. This basic flow is the solution of the 2D Navier-Stokes equations with periodic boundary conditions in two directions $0 \leq$ $x, y \leq 2 \pi$ given by

$$
\begin{aligned}
\overrightarrow{u_{t}}+(\vec{u} \cdot \nabla) \vec{u}+\nabla p & =\nu \nabla^{2} \vec{u}+\vec{f}, \\
\nabla \cdot \vec{u} & =0,
\end{aligned}
$$

with $\vec{u}=\left(u_{1}, u_{2}\right)$ and force $\vec{f}=\left(\alpha^{3} v \sin \alpha y, 0\right)$, which is assumed to be stationary and spatially biperiodic. The kinematic viscosity is $\nu=1 / R_{e}$, where $R_{e}$ is the Reynolds number and the pressure is $p$.

The perturbed nondimensional vorticity $\omega=-\Delta \phi^{\prime}$ formulation of the Kolmogorov flow is

$$
\begin{aligned}
\frac{\partial \Delta \phi^{\prime}}{\partial \tilde{t}}= & \Delta^{2} \phi^{\prime}-R_{e}\left[\frac{\partial}{\partial x}\left(\Delta \phi^{\prime} \frac{\partial \phi^{\prime}}{\partial y}\right)-\frac{\partial}{\partial y}\left(\Delta \phi^{\prime} \frac{\partial \phi^{\prime}}{\partial x}\right)\right] \\
& -\alpha R_{e} \sin \alpha y \frac{\partial}{\partial x}\left[\Delta \phi^{\prime}+\alpha^{2} \phi^{\prime}\right],
\end{aligned}
$$

where the scaled time $\tilde{t}=v t, u_{1}=\partial \phi / \partial y, u_{2}=-\partial \phi / \partial x$, and $\phi^{\prime}=\phi+\cos \alpha y$.

In Smaoui and Zribi [36-38], the derivation of the reduced order system of ODEs was based on expanding the stream function $\phi^{\prime}$ in (2) as follows:

$$
\begin{aligned}
\phi^{\prime}(x, y, t) & \\
= & \sum_{n=1}^{\infty} \phi_{0, n}^{\prime}(t) \cos (\alpha n y) \\
& +\sum_{m=1}^{2} \sum_{n=-\infty}^{\infty} \phi_{m, n}^{\prime}(t) \cos (m x+m y+\alpha n y) .
\end{aligned}
$$

In this paper, we use a totally different approach than the one used in [36-38]. We derive a system of seven ODEs from the Navier-Stokes equations by expanding $\vec{u}$ in the following form:

$$
\vec{u}(\mathbf{x})=\sum_{\mathbf{k} \neq \mathbf{0}} \exp (i \mathbf{k} \cdot \mathbf{x}) \gamma_{\mathbf{k}} \frac{\mathbf{k}^{\perp}}{|\mathbf{k}|},
$$

where $\mathbf{k}=\left(k_{1}, k_{2}\right)$ is a wave vector with integer components, $\mathbf{k}^{\perp}=\left(k_{2},-k_{1}\right)$, and the reality condition $\gamma_{\mathbf{k}}=-\bar{\gamma}_{-\mathbf{k}}$ must hold.

The equation for $\left\{\gamma_{\mathbf{k}}\right\}_{\mathbf{k} \neq \mathbf{0}}$ is

$$
\begin{aligned}
\dot{\gamma}_{\mathbf{k}}= & -i \sum_{\mathbf{k}_{1}+\mathbf{k}_{2}+\mathbf{k}=0} \frac{\left(\mathbf{k}_{1}^{\perp} \cdot \mathbf{k}_{2}\right)\left(k_{2}^{2}-k_{1}^{2}\right)}{2\left|\mathbf{k}_{1}\right|\left|\mathbf{k}_{2}\right||\mathbf{k}|} \bar{\gamma}_{\mathbf{k}_{1}} \bar{\gamma}_{\mathbf{k}_{2}}-\nu|\mathbf{k}|^{2} \gamma_{\mathbf{k}} \\
& +f_{\mathbf{k}},
\end{aligned}
$$

where $\gamma_{\mathbf{k}}=-\bar{\gamma}_{-\mathbf{k}}$ and $f_{\mathbf{k}}$ is the component of $\vec{f}$ with respect to $\left(\mathbf{k}^{\perp} /|\mathbf{k}|\right) \exp (i \mathbf{k} \cdot \mathbf{x})$.

Next, if the basic flow $\vec{u}=(2 \sin 2 y, 0)$, then one can consider the set of vectors, $\mathbf{k}_{\mathbf{1}}=(1,1), \mathbf{k}_{\mathbf{2}}=(0,3), \mathbf{k}_{\mathbf{3}}=(0,2)$, $\mathbf{k}_{\mathbf{4}}=(1,2), \mathbf{k}_{\mathbf{5}}=(0,1), \mathbf{k}_{\mathbf{6}}=(1,0)$, and $\mathbf{k}_{\mathbf{7}}=(1,-2)$, and their negatives in (5) to obtain the following system of ODEs:

$$
\begin{aligned}
& \dot{\gamma}_{\mathbf{k}_{1}=}=-i\left[\frac{4}{\sqrt{10}} \gamma_{\mathbf{k}_{4}} \bar{\gamma}_{\mathbf{k}_{5}}+\frac{-4}{\sqrt{10}} \gamma_{\mathbf{k}_{2}} \gamma_{\mathbf{k}_{7}}\right]-2 \nu \gamma_{\mathbf{k}_{1}}+f_{\mathbf{k}_{1}}, \\
& \dot{\gamma}_{\mathbf{k}_{2}}=-i\left[\frac{3}{\sqrt{10}} \gamma_{\mathbf{k}_{1}} \bar{\gamma}_{\mathbf{k}_{7}}\right]-9 v \gamma_{\mathbf{k}_{2}}+f_{\mathbf{k}_{2}}, \\
& \dot{\gamma}_{\mathbf{k}_{3}=}=-i\left[\frac{-4}{\sqrt{5}} \gamma_{\mathbf{k}_{4}} \bar{\gamma}_{\mathbf{k}_{6}}+\frac{4}{\sqrt{5}} \gamma_{\mathbf{k}_{6}} \bar{\gamma}_{\mathbf{k}_{7}}\right]-4 \nu \gamma_{\mathbf{k}_{3}}+f_{\mathbf{k}_{3}}, \\
& \dot{\gamma}_{\mathbf{k}_{4}=}=-i\left[\frac{1}{\sqrt{10}} \gamma_{\mathbf{k}_{1}} \gamma_{\mathbf{k}_{5}}+\frac{-3}{\sqrt{5}} \gamma_{\mathbf{k}_{3}} \gamma_{\mathbf{k}_{6}}\right]-5 \nu \gamma_{\mathbf{k}_{4}}+f_{\mathbf{k}_{4}}, \\
& \dot{\gamma}_{\mathbf{k}_{5}=}=-i\left[\frac{-3}{\sqrt{10}} \bar{\gamma}_{\mathbf{k}_{1}} \gamma_{\mathbf{k}_{4}}+\frac{-1}{\sqrt{2}} \gamma_{\mathbf{k}_{1}} \bar{\gamma}_{\mathbf{k}_{6}}\right]-\nu \gamma_{\mathbf{k}_{5}}+f_{\mathbf{k}_{5}}, \\
& \dot{\gamma}_{\mathbf{k}_{6}=}-i\left[\frac{1}{\sqrt{2}} \gamma_{\mathbf{k}_{1}} \bar{\gamma}_{\mathbf{k}_{5}}+\frac{1}{\sqrt{5}} \bar{\gamma}_{\mathbf{k}_{3}} \gamma_{\mathbf{k}_{4}}+\frac{1}{\sqrt{5}} \gamma_{\mathbf{k}_{3}} \gamma_{\mathbf{k}_{7}}\right]-\nu \gamma_{\mathbf{k}_{6}} \\
& +f_{\mathbf{k}_{6}}, \\
& \dot{\gamma}_{\mathbf{k}_{7}=}-i\left[\frac{-7}{\sqrt{10}} \gamma_{\mathbf{k}_{1}} \bar{\gamma}_{\mathbf{k}_{2}}+\frac{-3}{\sqrt{5}} \bar{\gamma}_{\mathbf{k}_{3}} \gamma_{\mathbf{k}_{6}}\right]-5 \nu \gamma_{\mathbf{k}_{7}}+f_{\mathbf{k}_{7}} .
\end{aligned}
$$


Let $\gamma_{\mathbf{k}_{1}}=i x_{1}, \gamma_{\mathbf{k}_{2}}=x_{2}, \gamma_{\mathbf{k}_{3}}=-i x_{3}, \gamma_{\mathbf{k}_{4}}=x_{4}, \gamma_{\mathbf{k}_{5}}=x_{5}$, $\gamma_{\mathbf{k}_{6}}=x_{6}$, and $\gamma_{\mathbf{k}_{7}}=x_{7}$; then system (6) becomes

$$
\begin{aligned}
& \dot{x}_{1}=\frac{-4}{\sqrt{10}} x_{4} x_{5}+\frac{4}{\sqrt{10}} x_{2} x_{7}-2 v x_{1}-i f_{\mathbf{k}_{1}}, \\
& \dot{x}_{2}=\frac{3}{\sqrt{10}} x_{1} x_{7}-9 v x_{2}+f_{\mathbf{k}_{2}}, \\
& \dot{x}_{3}=\frac{-4}{\sqrt{5}} x_{4} x_{6}+\frac{4}{\sqrt{5}} x_{6} x_{7}-4 v x_{3}+i f_{\mathbf{k}_{3}}, \\
& \dot{x}_{4}=\frac{1}{\sqrt{10}} x_{1} x_{5}+\frac{3}{\sqrt{5}} x_{3} x_{6}-5 v x_{4}+f_{\mathbf{k}_{4}}, \\
& \dot{x}_{5}=\frac{3}{\sqrt{10}} x_{1} x_{4}-\frac{1}{\sqrt{2}} x_{1} x_{6}-v x_{5}+f_{\mathbf{k}_{5}}, \\
& \dot{x}_{6}=\frac{1}{\sqrt{2}} x_{1} x_{5}+\frac{1}{\sqrt{5}} x_{3} x_{4}-\frac{1}{\sqrt{5}} x_{3} x_{7}-v x_{6}+f_{\mathbf{k}_{6}}, \\
& \dot{x}_{7}=\frac{-7}{\sqrt{10}} x_{1} x_{2}-\frac{3}{\sqrt{5}} x_{3} x_{6}-5 v x_{7}+f_{\mathbf{k}_{7}} .
\end{aligned}
$$

After changing the length scale $x_{i}=\sqrt{10} v \widetilde{x}_{i}$ for $i=$ $1, \ldots, 7$, the time scale $t=\widetilde{t} / v$ and $v=(\sqrt{10} / 5) \widetilde{\nu}$, and considering the forcing term to act on mode $\mathbf{k}_{\mathbf{3}}$, system (7) becomes

$$
\begin{aligned}
& \dot{x}_{1}=-2 x_{1}-4 x_{4} x_{5}+4 x_{2} x_{7}, \\
& \dot{x}_{2}=-9 x_{2}+3 x_{1} x_{7}, \\
& \dot{x}_{3}=-4 x_{3}-4 \sqrt{2} x_{4} x_{6}+4 \sqrt{2} x_{6} x_{7}+4 R_{e}, \\
& \dot{x}_{4}=-5 x_{4}+x_{1} x_{5}+3 \sqrt{2} x_{3} x_{6}, \\
& \dot{x}_{5}=-x_{5}+3 x_{1} x_{4}-\sqrt{5} x_{1} x_{6}, \\
& \dot{x}_{6}=-x_{6}+\sqrt{5} x_{1} x_{5}+\sqrt{2} x_{3} x_{4}-\sqrt{2} x_{3} x_{7}, \\
& \dot{x}_{7}=-5 x_{7}-7 x_{1} x_{2}-3 \sqrt{2} x_{3} x_{6} .
\end{aligned}
$$

The system given by the equations in (8) can be written in the following form:

$$
\dot{x}=A x+f(x),
$$

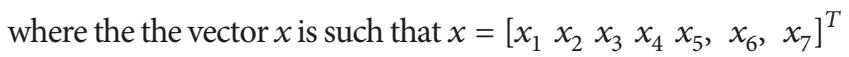
and the diagonal matrix $A$ is such that:

$$
A=\operatorname{diag}(-2,-9,-4,-5,-1,-1,-5) \text {, }
$$

and the nonlinear vector $f(x)$ is such that

$$
f=\left[\begin{array}{lllllll}
f_{1} & f_{2} & f_{3} & f_{4} & f_{5} & f_{6} & f_{7}
\end{array}\right]^{T}
$$

with

$$
\begin{aligned}
& f_{1}=-4 x_{4} x_{5}+4 x_{2} x_{7}, \\
& f_{2}=3 x_{1} x_{7}, \\
& f_{3}=-4 \sqrt{2} x_{4} x_{6}+4 \sqrt{2} x_{6} x_{7}+4 R_{e}, \\
& f_{4}=x_{1} x_{5}+3 \sqrt{2} x_{3} x_{6},
\end{aligned}
$$

$$
\begin{aligned}
& f_{5}=3 x_{1} x_{4}-\sqrt{5} x_{1} x_{6}, \\
& f_{6}=\sqrt{5} x_{1} x_{5}+\sqrt{2} x_{3} x_{4}-\sqrt{2} x_{3} x_{7}, \\
& f_{7}=-7 x_{1} x_{2}-3 \sqrt{2} x_{3} x_{6} .
\end{aligned}
$$

Remark 1. It is noted that system (8) is invariant under the following symmetries:

$$
\begin{gathered}
r_{x}:\left(x_{1}, x_{2}, x_{3}, x_{4}, x_{5}, x_{6}, x_{7}\right) \Longleftrightarrow \\
\left(x_{1},-x_{2}, x_{3},-x_{4},-x_{5},-x_{6},-x_{7}\right), \\
r_{y}:\left(x_{1}, x_{2}, x_{3}, x_{4}, x_{5}, x_{6}, x_{7}\right) \Longleftrightarrow \\
\left(-x_{1},-x_{2}, x_{3}, x_{4},-x_{5}, x_{6}, x_{7}\right), \\
r_{o}:\left(x_{1}, x_{2}, x_{3}, x_{4}, x_{5}, x_{6}, x_{7}\right) \Longleftrightarrow \\
\left(-x_{1}, x_{2}, x_{3},-x_{4}, x_{5},-x_{6},-x_{7}\right),
\end{gathered}
$$

where $r_{x}, r_{y}$, and $r_{o}$ are reflection symmetries across the $x$ axis, the $y$-axis, and the origin, respectively. Hence, $r_{x}, r_{y}, r_{o}$ with the identity transformation $i$ form an Abelian group: $G=$ $\left\{r_{x}, r_{y}, r_{o}, i\right\}$.

2.2. The Dynamics of the Seven-Mode Truncation System. In this subsection, we analyze the dynamics of the sevenmode truncation ODE system presented in (8) for different Reynolds numbers. The DsTool software [40] is used in all numerical simulations presented in this section using the 4 thorder Runge-Kutta method as the numerical integrator and with the time step $\Delta t=0.0001$.

(i) For $0 \leq R_{e} \leq R_{1} \simeq 0.645$, the basic fixed point $P_{0}=\left(0,0, R_{e}, 0,0,0,0\right)$ is the only stable solution and, numerically, it is globally attractive for all $R_{e} \leq R_{1}$. This is a special case of the general results on the theory of Navier-Stokes equations [41].

(ii) For $R_{1}<R_{e} \leq R_{2} \simeq 2.469$ there are three fixed points: the old one $P_{0}$, which becomes unstable as a result of the crossing of the imaginary axes by one of the eigenvalues of the Lyapunov matrix, and two more, $P_{1}$ and $P_{2}$, that bifurcated from $P_{0}$ are stable and attracting. Numerical evidence shows that any randomly chosen initial condition is either attracted by $P_{1}$ or $P_{2}$.

(iii) For $R_{2}<R_{e} \leq R_{3} \simeq 20.844$, there are seven fixed points: four asymptotically stable and three unstable. Figure 1 presents the phase portrait of the four asymptotically stable fixed points at $R_{e}=18$. The first asymptotically state fixed point (Figure 1 , top left) was obtained using the following initial conditions: 


$$
\left[\begin{array}{lllllll}
x_{1} & x_{2} & x_{3} & x_{4} & x_{5} & x_{6} & x_{7}
\end{array}\right]^{T}=\left[\begin{array}{c}
-0.8275 \\
-0.4703 \\
0.7234 \\
-2.3355 \\
-0.1220 \\
-3.5990 \\
0.4255
\end{array}\right] \text {. }
$$

The second asymptotically stable fixed point (Figure 1, bottom right) was obtained by applying the reflection symmetry $r_{o}$ on the initial conditions used for the first asymptotically stable fixed point. That is,

$$
\left[\begin{array}{lllllll}
x_{1} & x_{2} & x_{3} & x_{4} & x_{5} & x_{6} & x_{7}
\end{array}\right]^{T}=r_{o}\left[\begin{array}{c}
-0.8275 \\
-0.4703 \\
0.7234 \\
-2.3355 \\
-0.1220 \\
-3.5990 \\
0.4255
\end{array}\right]
$$

$$
=\left[\begin{array}{c}
0.8275 \\
-0.4703 \\
0.7234 \\
2.3355 \\
-0.1220 \\
3.5990 \\
-0.4255
\end{array}\right]
$$

The third asymptotically stable fixed point (Figure 1, top right) was generated by applying the reflection symmetry $r_{x}$ on the initial conditions used for the second asymptotically stable fixed point. That is,

$$
\left[\begin{array}{lllllll}
x_{1} & x_{2} & x_{3} & x_{4} & x_{5} & x_{6} & x_{7}
\end{array}\right]^{T}=r_{x}\left[\begin{array}{c}
0.8275 \\
-0.4703 \\
0.7234 \\
2.3355 \\
-0.1220 \\
3.5990 \\
-0.4255
\end{array}\right]
$$$$
=\left[\begin{array}{c}
0.8275 \\
0.4703 \\
0.7234 \\
-2.3355 \\
0.1220 \\
-3.5990 \\
0.4255
\end{array}\right]
$$

The fourth asymptotically stable fixed point (Figure 1, bottom left) was obtained by applying the reflection symmetry $r_{y}$ on the initial conditions used for the second asymptotically stable fixed point. That is,

$\left[\begin{array}{lllllll}x_{1} & x_{2} & x_{3} & x_{4} & x_{5} & x_{6} & x_{7}\end{array}\right]^{T}=r_{y}\left[\begin{array}{c}0.8275 \\ -0.4703 \\ 0.7234 \\ 2.3355 \\ -0.1220 \\ 3.5990 \\ -0.4255\end{array}\right]$

$$
=\left[\begin{array}{c}
-0.8275 \\
0.4703 \\
0.7234 \\
2.3355 \\
0.1220 \\
3.5990 \\
-0.4255
\end{array}\right] .
$$

(iv) For $R_{3}<R_{e} \leq R_{4} \simeq 26.323$ the four asymptotically stable fixed points become unstable because a pair of complex eigenvalues crosses the imaginary axes, and bifurcate via a Hopf bifurcation into a four stable periodic orbits around these four fixed points. The four stable periodic orbits remain stable up to $R_{e}=$ $R_{4}=26.323$. The other three remain unstable (see Figure 2).

(v) For $R_{4}<R_{e} \leq R_{5} \simeq 26.375$. At $R_{e}=R_{4}=26.323$ each of the four periodic orbits loses stability and bifurcates into a new periodic orbit with double the period.

(vi) For $R_{5}<R_{e} \leq R_{6} \simeq 26.41$, a homoclinic gluing bifurcation similar to the one observed by Armbruster et al. [25] occurs (see Figure 3). It should be noted that the homoclinic gluing bifurcation observed leads to two chaotic or strange attractors each connecting a pair of two stable periodic orbits. Figure 3(b) shows that the dynamics of one of the chaotic attractors follow a quasiperiodic regime; then either bursts to the same attractor or to its symmetric counterpart; then other bursts follow. Numerical simulations indicate that intervals between bursts become shorter as the Reynolds number, $R_{e}$, is increased up to $R_{e}=R_{6}$.

\section{Controlling the Dynamics of the Reduced Order System to a Fixed Point}

This section deals with the design of a control scheme to drive the states of the system to a stable or unstable desired fixed point. Hence, control inputs are added to the system in (8). The controlled system of ODEs with control inputs added to 


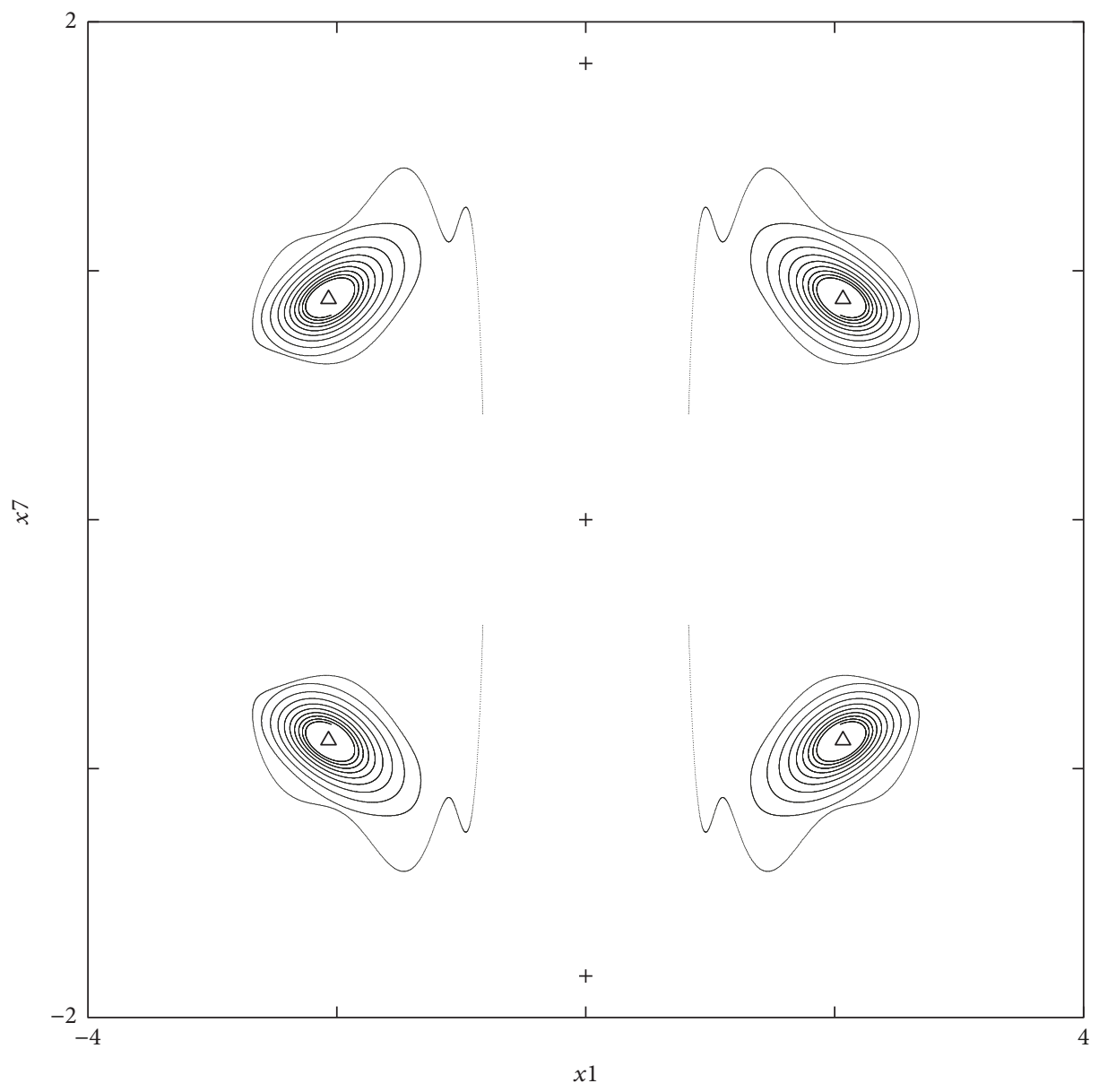

FIGURE 1: The phase portrait of the four asymptotically stable fixed points at $R_{e}=18$; the first asymptotically stable fixed point (top left) is obtained using the following initial conditions: $\left(x_{1}, x_{2}, x_{3}, x_{4}, x_{5}, x_{6}, x_{7}\right)=(-0.8275,-0.4703,0.7234,-2.3355,-0.122,-3.599,0.4255)$; the second asymptotically stable fixed point (bottom right) is obtained using the initial conditions: $\left(x_{1}, x_{2}, x_{3}, x_{4}, x_{5}, x_{6}, x_{7}\right)=(0.8275,-0.4703$, $0.7234,2.3355,-0.122,3.599,-0.4255)=r_{o}(-0.8275,-0.4703,0.7234,-2.3355,-0.122,-3.599,0.4255)$; the third asymptotically stable fixed point (top right) is generated using the initial conditions: $\left(x_{1}, x_{2}, x_{3}, x_{4}, x_{5}, x_{6}, x_{7}\right)=(0.8275,0.4703,0.7234,-2.3355,0.122,-3.599,0.4255)$ $=r_{x}(0.8275,-0.4703,0.7234,2.3355,-0.122,3.599,-0.4255)$; the fourth asymptotically stable fixed point (bottom left) is generated using the initial conditions: $\left(x_{1}, x_{2}, x_{3}, x_{4}, x_{5}, x_{6}, x_{7}\right)=(-0.8275,0.4703,0.7234,2.3355,0.122,3.599,-0.4255)=r_{y}(0.8275,-0.4703,0.7234,2.3355$, $-0.122,3.599,-0.4255)$.

the first, the third, the fifth, and the sixth ODE of (8) is as follows.

$$
\begin{aligned}
& \dot{x}_{1}=-2 x_{1}-4 x_{4} x_{5}+4 x_{2} x_{7}+u_{1}, \\
& \dot{x}_{2}=-9 x_{2}+3 x_{1} x_{7}, \\
& \dot{x}_{3}=-4 x_{3}-4 \sqrt{2} x_{4} x_{6}+4 \sqrt{2} x_{6} x_{7}+4 R_{e}+u_{2}, \\
& \dot{x}_{4}=-5 x_{4}+x_{1} x_{5}+3 \sqrt{2} x_{3} x_{6}, \\
& \dot{x}_{5}=-x_{5}+3 x_{1} x_{4}-\sqrt{5} x_{1} x_{6}+u_{3}, \\
& \dot{x}_{6}=-x_{6}+\sqrt{5} x_{1} x_{5}+\sqrt{2} x_{3} x_{4}-\sqrt{2} x_{3} x_{7}+u_{4}, \\
& \dot{x}_{7}=-5 x_{7}-7 x_{1} x_{2}-3 \sqrt{2} x_{3} x_{6} .
\end{aligned}
$$

Let the constant desired fixed point be such that $x_{d}=$ $\left[\begin{array}{llllllll}x_{1 d} & x_{2 d} & x_{3 d} & x_{4 d} & x_{5 d} & x_{6 d} & x_{7 d}\end{array}\right]^{T}$. Since $x_{d}$ is an equilibrium point of system (18) then it must satisfy the following algebraic equations:

$$
\begin{array}{r}
-9 x_{2 d}+3 x_{1 d} x_{7 d}=0 \\
-5 x_{4 d}+x_{1 d} x_{5 d}+3 \sqrt{2} x_{3 d} x_{6 d}=0 \\
-5 x_{7 d}-7 x_{1 d} x_{2 d}-3 \sqrt{2} x_{3 d} x_{6 d}=0 .
\end{array}
$$

Define the errors $e_{i}(i=1, \ldots, 7)$ such that

$$
\begin{aligned}
& e_{1}=x_{1}-x_{1 d}, \\
& e_{2}=x_{2}-x_{2 d}, \\
& e_{3}=x_{3}-x_{3 d}, \\
& e_{4}=x_{4}-x_{4 d},
\end{aligned}
$$


6

Complexity

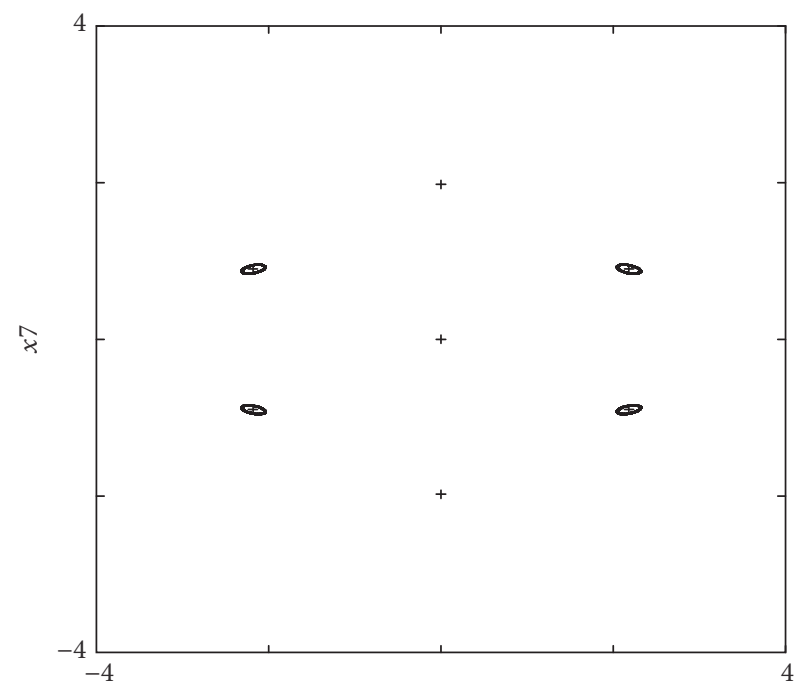

$x 1$

(a) $R_{e}=20.845$

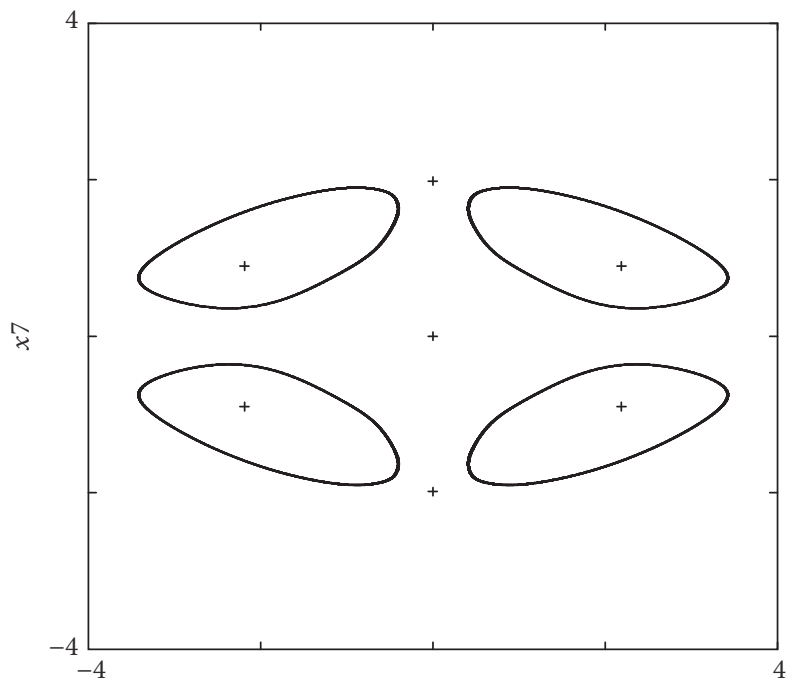

$x 1$

(c) $R_{e}=25$

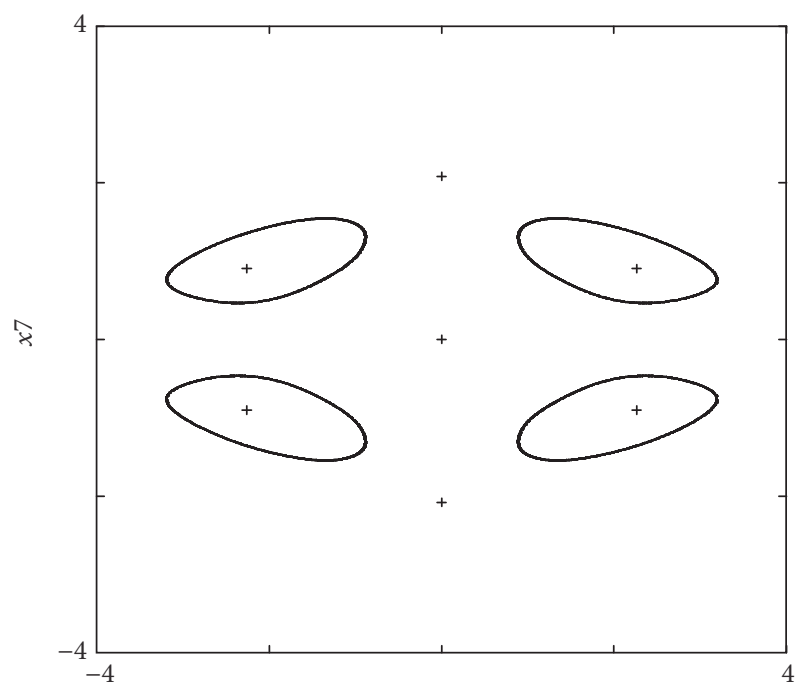

$x 1$

(b) $R_{e}=23$

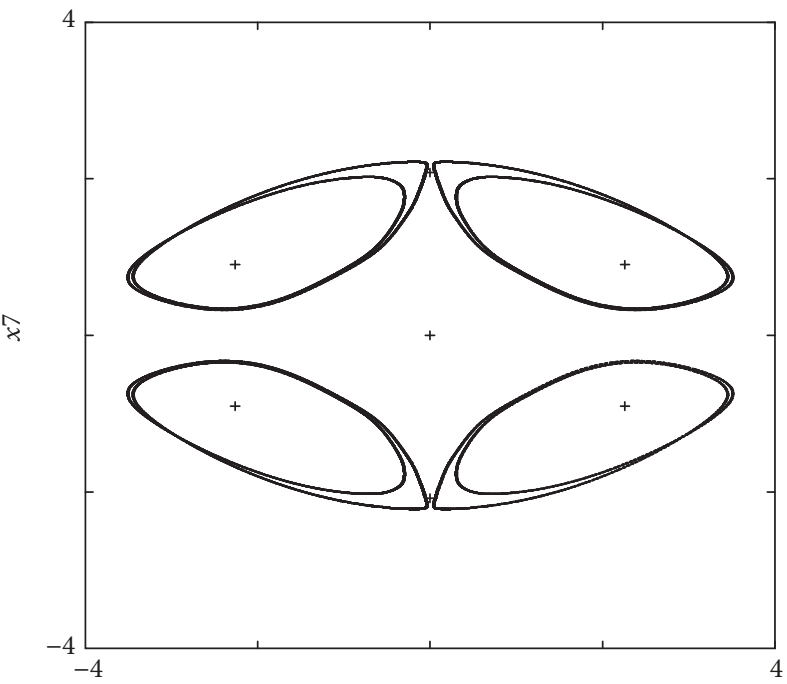

$x 1$

(d) $R_{e}=26.37$

FIGURE 2: Phase portraits of the four stable periodic orbits arising from a Hope bifurcation at $R_{e}=20.845$ that remain stable for up to $R_{e}=26.323$ and bifurcates again into a new four periodic orbits with double the period.

$$
\begin{aligned}
& e_{5}=x_{5}-x_{5 d} \\
& e_{6}=x_{6}-x_{6 d} \\
& e_{7}=x_{7}-x_{7 d} .
\end{aligned}
$$

Using equations (18), (19), and (20), the model for the error system can be written as follows:

$$
\begin{aligned}
\dot{e}_{1}= & -2 e_{1}-4 e_{4} e_{5}-4 x_{5 d} e_{4}-4 x_{4 d} e_{5}+4 e_{2} e_{7}+4 x_{7 d} e_{2} \\
& +4 x_{2 d} e_{7}+m_{1}+u_{1}, \\
\dot{e}_{2}= & -9 e_{2}+3 e_{1} e_{7}+3 x_{7 d} e_{1}+3 x_{1 d} e_{7},
\end{aligned}
$$

$$
\begin{aligned}
\dot{e}_{3}= & -4 e_{3}-4 \sqrt{2} e_{4} e_{6}-4 \sqrt{2} x_{6 d} e_{4}-4 \sqrt{2} x_{4 d} e_{6} \\
& +4 \sqrt{2} e_{6} e_{7}+4 \sqrt{2} x_{7 d} e_{6}+4 \sqrt{2} x_{6 d} e_{7}+m_{2} \\
& +u_{2}, \\
\dot{e}_{4}= & -5 e_{4}+e_{1} e_{5}+x_{5 d} e_{1}+x_{1 d} e_{5}+3 \sqrt{2} e_{3} e_{6} \\
& +3 \sqrt{2} x_{3 d} e_{6}+3 \sqrt{2} x_{6 d} e_{3}, \\
\dot{e}_{5}= & -e_{5}+3 e_{1} e_{4}+3 x_{1 d} e_{4}+3 x_{4 d} e_{1}-\sqrt{5} e_{1} e_{6} \\
& -\sqrt{5} x_{6 d} e_{1}-\sqrt{5} x_{1 d} e_{6}+m_{3}+u_{3}, \\
\dot{e}_{6}= & -e_{6}+\sqrt{5} e_{1} e_{5}+\sqrt{5} x_{5 d} e_{1}+\sqrt{5} x_{1 d} e_{5}+\sqrt{2} e_{3} e_{4}
\end{aligned}
$$




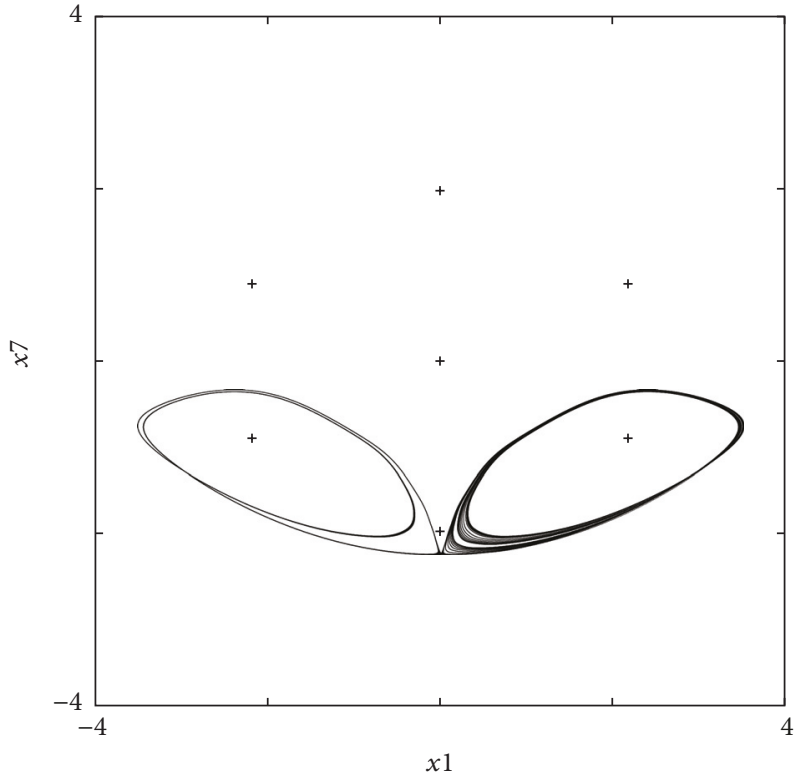

(a) $R_{e}=26.377$

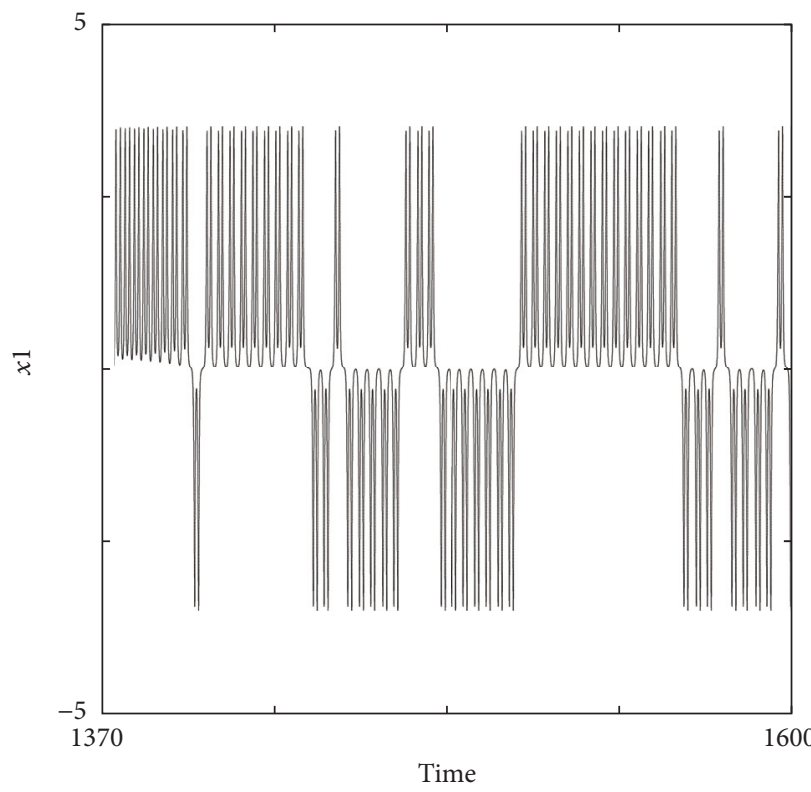

(b) $R_{e}=26.377$

FIgURE 3: (a) Phase portraits of the bursting phenomena resulting from a homoclinic gluing bifurcation connecting a pair of two stable periodic orbits. (b) Time series of the state $x_{1}$ versus time at $R_{e}=26.377$.

$$
\begin{aligned}
& +\sqrt{2} x_{3 d} e_{4}+\sqrt{2} x_{4 d} e_{3}-\sqrt{2} e_{3} e_{7}-\sqrt{2} x_{3 d} e_{7} \\
& -\sqrt{2} x_{7 d} e_{3}+m_{4}+u_{4}, \\
\dot{e}_{7}= & -5 e_{7}-7 e_{1} e_{2}-7 x_{2 d} e_{1}-7 x_{1 d} e_{2}-3 \sqrt{2} e_{3} e_{6} \\
& -3 \sqrt{2} x_{6 d} e_{3}-3 \sqrt{2} x_{3 d} e_{6},
\end{aligned}
$$

where the parameters $m_{1}, m_{2}, m_{3}$, and $m_{4}$ are such that

$$
\begin{aligned}
& m_{1}=-2 x_{1 d}-4 x_{4 d} x_{5 d}+4 x_{2 d} x_{7 d}, \\
& m_{2}=4 R_{e}-4 x_{3 d}-4 \sqrt{2} x_{4 d} x_{6 d}+4 \sqrt{2} x_{6 d} x_{7 d}, \\
& m_{3}=-x_{5 d}+3 x_{1 d} x_{4 d}-\sqrt{5} x_{1 d} x_{6 d}, \\
& m_{4}=-x_{6 d}+\sqrt{5} x_{1 d} x_{5 d}+\sqrt{2} x_{3 d} x_{4 d}-\sqrt{2} x_{3 d} x_{7 d} .
\end{aligned}
$$

Let the gains $\overline{c_{i}}(i=1, \ldots, 4)$ be positive scalars and let the control gains $a_{i}(i=1, \ldots, 7)$ be positive scalars such that

$$
3 a_{2}-7 a_{7}=0
$$

Theorem 2. The control law,

$$
\begin{aligned}
u_{1}= & 4 e_{4} e_{5}+4 x_{5 d} e_{4}+4 x_{4 d} e_{5}-4 e_{2} e_{7}-4 x_{7 d} e_{2} \\
& -4 x_{2 d} e_{7}-3 \frac{a_{2}}{a_{1}} e_{2} e_{7}-3 \frac{a_{2}}{a_{1}} x_{7 d} e_{2}-\frac{a_{4}}{a_{1}} e_{4} e_{5} \\
& -\frac{a_{4}}{a_{1}} x_{5 d} e_{4}+7 \frac{a_{7}}{a_{1}} e_{2} e_{7}+7 \frac{a_{7}}{a_{1}} x_{2 d} e_{7}-m_{1} \\
& -\bar{c}_{1} e_{1},
\end{aligned}
$$

$$
\begin{aligned}
u_{2}= & 4 \sqrt{2} e_{4} e_{6}+4 \sqrt{2} x_{4 d} e_{6}+4 \sqrt{2} x_{6 d} e_{4}-4 \sqrt{2} e_{6} e_{7} \\
& -4 \sqrt{2} x_{6 d} e_{7}-4 \sqrt{2} x_{7 d} e_{6}-3 \sqrt{2} \frac{a_{4}}{a_{3}} e_{4} e_{6} \\
& -3 \sqrt{2} \frac{a_{4}}{a_{3}} x_{6 d} e_{4}+3 \sqrt{2} \frac{a_{7}}{a_{3}} e_{6} e_{7}+3 \sqrt{2} \frac{a_{7}}{a_{3}} x_{6 d} e_{7} \\
& -m_{2}-\overline{c_{2}} e_{3}, \\
u_{3}= & -3 e_{1} e_{5}-3 x_{1 d} e_{4}-3 x_{4 d} e_{1}+\sqrt{5} e_{1} e_{6}+\sqrt{5} x_{1 d} e_{6} \\
& +\sqrt{5} x_{6 d} e_{1}-\frac{a_{4}}{a_{5}} x_{1 d} e_{4}-m_{3}-\overline{c_{3}} e_{5}, \\
u_{4}= & -\sqrt{5} e_{1} e_{5}-\sqrt{5} x_{5 d} e_{1}-\sqrt{5} x_{1 d} e_{5}-\sqrt{2} e_{3} e_{4} \\
& -\sqrt{2} x_{3 d} e_{4}-\sqrt{2} x_{4 d} e_{3}+\sqrt{2} e_{3} e_{7}+\sqrt{2} x_{3 d} e_{7} \\
& +\sqrt{2} x_{7 d} e_{3}-3 \sqrt{2} \frac{a_{4}}{a_{6}} x_{3 d} e_{4}+3 \sqrt{2} \frac{a_{7}}{a_{6}} x_{3 d} e_{7} \\
& -m_{4}-\overline{c_{4}} e_{6},
\end{aligned}
$$

when applied to the error model (21) guarantees the convergence of the errors $e_{i}(i=1, \ldots, 7)$ to zero as t tends to infinity. Therefore, system (18) asymptotically converges to a desired fixed point $x_{d}$ as t tends to infinity.

Proof. Consider the Lyapunov function candidate $V$ such that

$$
\begin{aligned}
V= & \frac{1}{2} a_{1} e_{1}^{2}+\frac{1}{2} a_{2} e_{2}^{2}+\frac{1}{2} a_{3} e_{3}^{2}+\frac{1}{2} a_{4} e_{4}^{2}+\frac{1}{2} a_{5} e_{5}^{2} \\
& +\frac{1}{2} a_{6} e_{6}^{2}+\frac{1}{2} a_{7} e_{7}^{2} .
\end{aligned}
$$


Using the model of the errors system given in (21), the control law given by (24), and the constraints on the control parameters $a_{2}$ and $a_{7}$ given by (23), the derivative of $V$ with respect to time is such that

$$
\begin{aligned}
\dot{V}= & -2 a_{1} e_{1}^{2}-9 a_{2} e_{2}^{2}-4 a_{3} e_{3}^{2}-5 a_{4} e_{4}^{2}-a_{5} e_{5}^{2}-a_{6} e_{6}^{2} \\
& -5 a_{7} e_{7}^{2}-\overline{c_{1}} a_{1} e_{1}^{2}-\overline{c_{2}} a_{3} e_{3}^{2}-\overline{c_{3}} a_{5} e_{5}^{2}-\overline{c_{4}} a_{6} e_{6}^{2} .
\end{aligned}
$$

Since the design parameters $a_{i}(i=1, \ldots, 7)$ and $\overline{c_{i}}(i=$ $1, \ldots, 4)$ are positive constants, then it can be concluded that $\dot{V}$ is negative definite. Therefore, the Lyapunov function $V$ in (25) is positive definite, its derivative with respect to time $\dot{V}$ is negative definite, and $V$ is radially unbounded. Hence, it can be concluded that the errors $e_{i}(t)(i=1, \ldots, 7)$ in (20) asymptotically converge to zero as $t$ tends to infinity. Thus, the states of system (18) asymptotically converge to a desired constant fixed point $x_{d}$ as $t$ tends to infinity.

The simulation results of system (18) when controlled using the proposed control scheme given by (24) are presented below. The simulations are carried out for the Reynolds number $R_{e}=26$. Note that the dynamics at $R_{e}=26$ corresponds to four stable periodic solutions of the system of ODEs. These periodic solutions are a result of Hopf bifurcations that took place at $R_{e}=20.845$. The values of the control gains $\overline{c_{i}}$ are such that $\overline{c_{1}}=10, \overline{c_{2}}=10, \overline{c_{3}}=10$, and $\overline{c_{4}}=10$. The control gains $a_{1}-a_{7}$ are taken to be $a_{1}=1, a_{2}=7$, $a_{3}=1, a_{4}=1, a_{5}=1, a_{6}=1$, and $a_{7}=3$. The simulation results correspond to an initial condition,

$$
x(0)=\left[\begin{array}{lllllll}
-2.26043 & 0.68026 & 0.893857 & 2.9772 & -0.17333 & 4.0286 & -0.90282
\end{array}\right]^{T},
$$

and the four desired fixed points

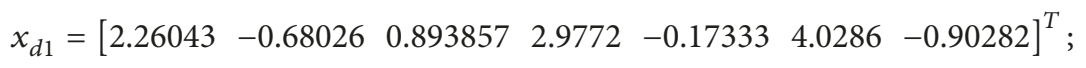

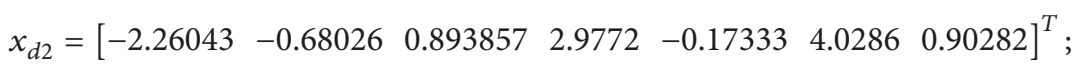

$$
\begin{aligned}
& x_{d 3}=\left[\begin{array}{lllllll}
-2.26043 & -0.68026 & 0.893857 & -2.9772 & -0.17333 & -4.0286 & 0.90282
\end{array}\right]^{T} \text {; }
\end{aligned}
$$

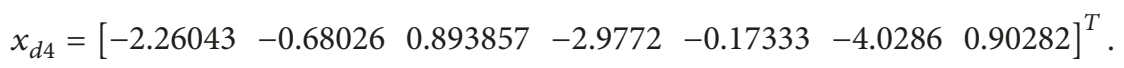

These desired fixed points are the asymptotically stable fixed points that became unstable as the result of Hopf bifurcations. At the beginning of the simulations, the controllers $u_{i}(i=$ $1, \ldots, 4)$ are set to zero for the first $200,000 \Delta t$. Then the control law given by (24) is switched on and off and the desired fixed point $x_{d}$ is set to one of the four desired fixed points each time the control law is switched on.

The simulation results are presented in Figures 4 and 5. Figure 4 presents the controlled states $x_{7}(t)$ versus $x_{1}(t)$, and Figure 5 depicts the time evolution of the controlled states $x_{i}(t)(i=1, \ldots, 7)$. The figures clearly show how the controlled states $x_{i}(t)$ converge to one of the desired fixed points each time the control law is switched on and to one of the four stable periodic solutions when the control law is switched off. Hence, it can be concluded that the proposed controller given by (24) drives the states of the system in (18) to their desired states.

\section{Synchronization of Two ODE Systems Having the Same or Different Reynolds Numbers}

In this section, we design a control law to synchronize two ODE systems obtained from the 2D Navier-Stokes equation using the Fourier Expansion truncation method having the same or different Reynolds numbers.

4.1. Model of the Error System. In this subsection, we first derive the error system between the two ODE systems to be synchronized. The model of the first ODE system is

$$
\begin{aligned}
& \dot{x}_{1}=-2 x_{1}-4 x_{4} x_{5}+4 x_{2} x_{7}, \\
& \dot{x}_{2}=-9 x_{2}+3 x_{1} x_{7}, \\
& \dot{x}_{3}=-4 x_{3}-4 \sqrt{2} x_{4} x_{6}+4 \sqrt{2} x_{6} x_{7}+4 R_{e 1}, \\
& \dot{x}_{4}=-5 x_{4}+x_{1} x_{5}+3 \sqrt{2} x_{3} x_{6}, \\
& \dot{x}_{5}=-x_{5}+3 x_{1} x_{4}-\sqrt{5} x_{1} x_{6}, \\
& \dot{x}_{6}=-x_{6}+\sqrt{5} x_{1} x_{5}+\sqrt{2} x_{3} x_{4}-\sqrt{2} x_{3} x_{7}, \\
& \dot{x}_{7}=-5 x_{7}-7 x_{1} x_{2}-3 \sqrt{2} x_{3} x_{6},
\end{aligned}
$$

where $R_{e 1}$ is the Reynolds number of the first ODE system. The model of the second ODEs system is

$$
\begin{aligned}
& \dot{y}_{1}=-2 y_{1}-4 y_{4} y_{5}+4 y_{2} y_{7}+u_{1}, \\
& \dot{y}_{2}=-9 y_{2}+3 y_{1} y_{7},
\end{aligned}
$$




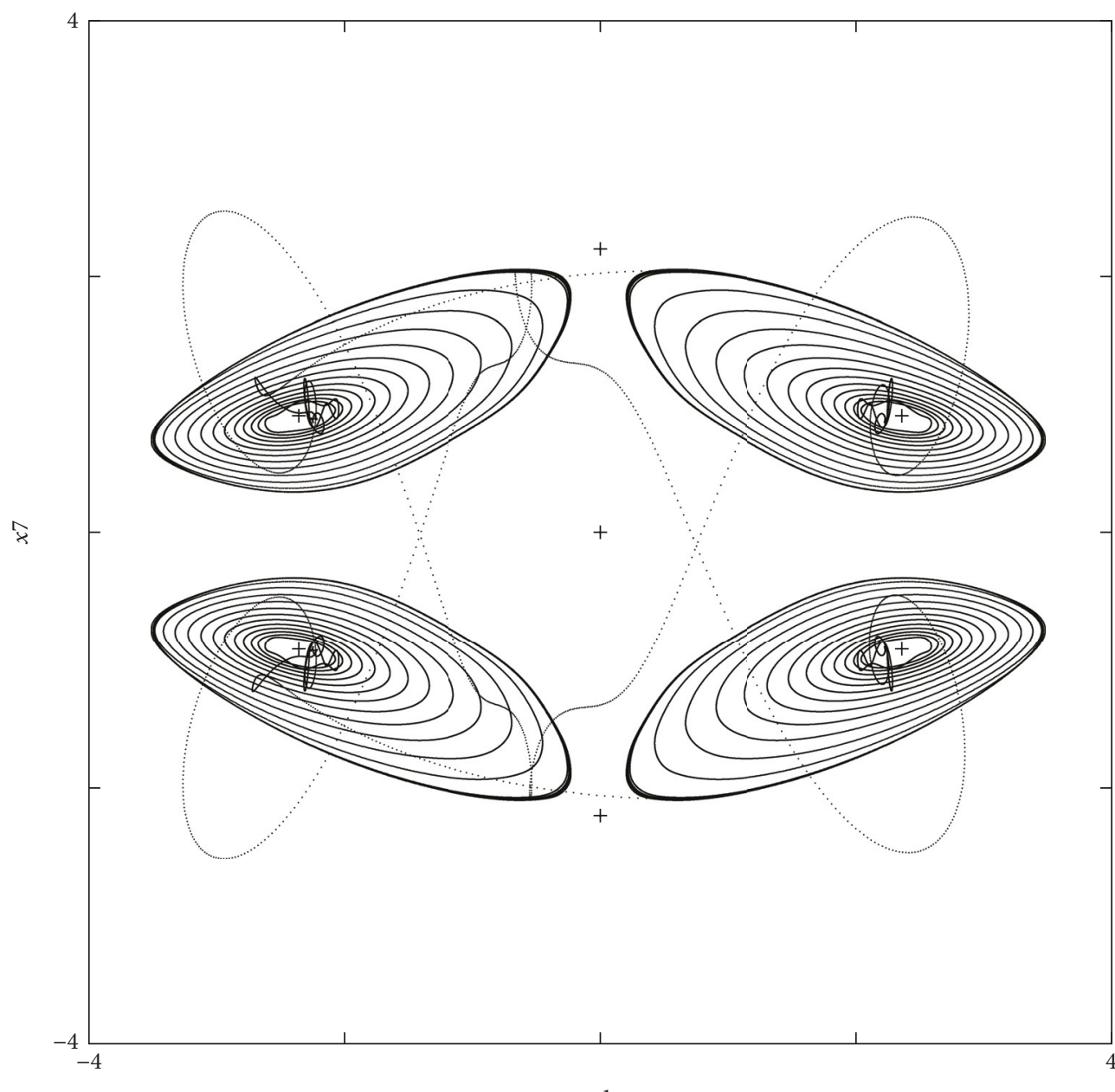

$x 1$

Figure 4: The state $x_{7}$ versus $x_{1}$ at $R_{e}=26$ when the controllers are switched on and off and when the desired fixed point is one of the four unstable fixed points.

$$
\begin{aligned}
& \dot{y}_{3}=-4 y_{3}-4 \sqrt{2} y_{4} y_{6}+4 \sqrt{2} y_{6} y_{7}+4 R_{e 2}+u_{2}, \\
& \dot{y}_{4}=-5 y_{4}+y_{1} y_{5}+3 \sqrt{2} y_{3} y_{6}, \\
& \dot{y}_{5}=-y_{5}+3 y_{1} y_{4}-\sqrt{5} y_{1} y_{6}+u_{3}, \\
& \dot{y}_{6}=-y_{6}+\sqrt{5} y_{1} y_{5}+\sqrt{2} y_{3} y_{4}-\sqrt{2} y_{3} y_{7}+u_{4}, \\
& \dot{y}_{7}=-5 y_{7}-7 y_{1} y_{2}-3 \sqrt{2} y_{3} y_{6},
\end{aligned}
$$

where $y_{i}(i=1, \ldots, 7)$ are the state variables of the second $\mathrm{ODE}$ and $R_{e 2}$ is its Reynolds number. Define the errors $e_{i}(t)(i=1, \ldots, 7)$ between the first ODE system in (29) and the second ODE system in (30) as follows:

$$
\begin{aligned}
& e_{1}=x_{1}-y_{1}, \\
& e_{2}=x_{2}-y_{2}, \\
& e_{3}=x_{3}-y_{3}, \\
& e_{4}=x_{4}-y_{4},
\end{aligned}
$$

$$
\begin{aligned}
& e_{5}=x_{5}-y_{5}, \\
& e_{6}=x_{6}-y_{6}, \\
& e_{7}=x_{7}-y_{7} .
\end{aligned}
$$

Using the equations defined in (29)-(31), the model of the error system can be written as follows:

$$
\begin{aligned}
\dot{e}_{1}= & -2 e_{1}-4 e_{4} e_{5}-4 y_{5} e_{4}-4 y_{4} e_{5}+4 e_{2} e_{7}+4 y_{7} e_{2} \\
& +4 y_{2} e_{7}-u_{1}, \\
\dot{e}_{2}= & -9 e_{2}+3 e_{1} e_{7}+3 y_{7} e_{1}+3 y_{1} e_{7}, \\
\dot{e}_{3}= & -4 e_{3}-4 \sqrt{2} e_{4} e_{6}-4 \sqrt{2} y_{6} e_{4}-4 \sqrt{2} y_{4} e_{6} \\
& +4 \sqrt{2} e_{6} e_{7}+4 \sqrt{2} y_{7} e_{6}+4 \sqrt{2} y_{6} e_{7}+4 R-u_{2}, \\
\dot{e}_{4}= & -5 e_{4}+e_{1} e_{5}+y_{5} e_{1}+y_{1} e_{5}+3 \sqrt{2} e_{3} e_{6}+3 \sqrt{2} y_{6} e_{3} \\
& +3 \sqrt{2} y_{3} e_{6}, \\
\dot{e}_{5}= & -e_{5}+3 e_{1} e_{4}+3 y_{4} e_{1}+3 y_{1} e_{4}-\sqrt{5} e_{1} e_{6}-\sqrt{5} y_{6} e_{1}
\end{aligned}
$$



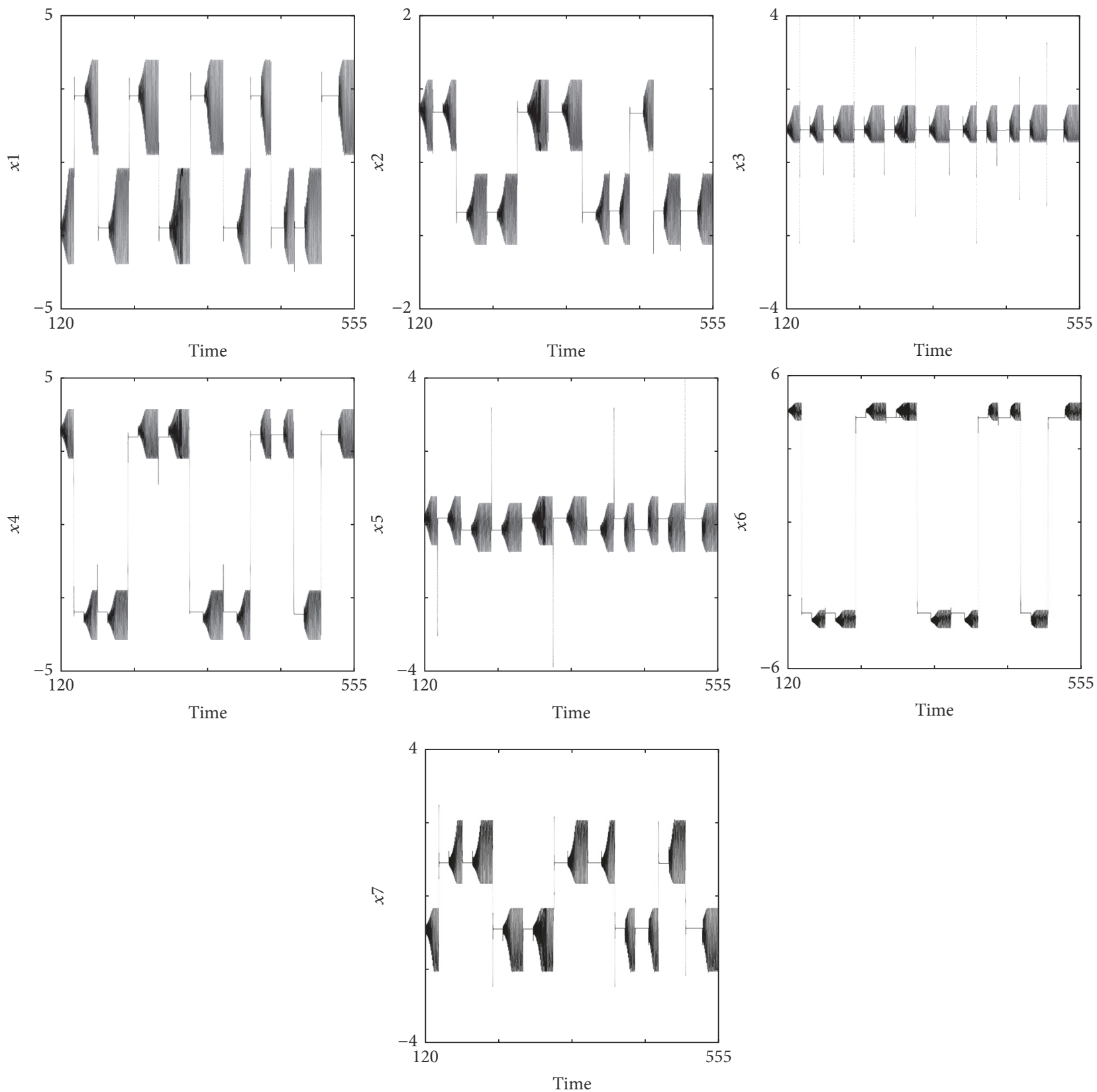

FIGURE 5: The states $x_{i}(i=1, \ldots, 7)$ versus time at $R_{e}=26$ when the controllers are switched on and off and when the desired fixed point is one of the four unstable fixed points.

$$
\begin{aligned}
& -\sqrt{5} y_{1} e_{6}-u_{3}, \\
\dot{e}_{6}= & -e_{6}+\sqrt{5} e_{1} e_{5}+\sqrt{5} y_{5} e_{1}+\sqrt{5} y_{1} e_{5}+\sqrt{2} e_{3} e_{4} \\
& +\sqrt{2} y_{4} e_{3}+\sqrt{2} y_{3} e_{4}-\sqrt{2} e_{3} e_{7}-\sqrt{2} y_{7} e_{3} \\
& -\sqrt{2} y_{3} e_{7}-u_{4}, \\
\dot{e}_{7}= & -5 e_{7}-7 e_{1} e_{2}-7 y_{2} e_{1}-7 y_{1} e_{2}-3 \sqrt{2} e_{3} e_{6} \\
& -3 \sqrt{2} y_{6} e_{3}-3 \sqrt{2} y_{3} e_{6},
\end{aligned}
$$

where $R=R_{e 1}-R_{e 2}$.
4.2. A Lyapunov Based Controller to Synchronize Two Systems Having the Same or Different Reynolds Number. In this subsection, a Lyapunov based controller is designed to drive the states of the system in (30) to asymptotically converge to the states of the system in (29).

Let the gains $g_{i}(i=1, \ldots, 4)$ be positive scalar. In addition, let the control gains $b_{i}(i=1, \ldots, 7)$ be positive scalars such that

$$
3 b_{2}-7 b_{7}=0
$$

The following theorem gives the second control result of the paper. 
Theorem 3. The control law,

$$
\begin{aligned}
u_{1}= & -4 e_{4} e_{5}-4 y_{5} e_{4}-4 y_{4} e_{5}+4 e_{2} e_{7}+4 y_{7} e_{2}+4 y_{2} e_{7} \\
& +3 \frac{b_{2}}{b_{1}} e_{2} e_{7}+3 \frac{b_{2}}{b_{1}} y_{7} e_{2}+\frac{b_{4}}{b_{1}} e_{4} e_{5}+\frac{b_{4}}{b_{1}} y_{5} e_{4} \\
& -7 \frac{b_{7}}{b_{1}} e_{2} e_{7}-7 \frac{b_{7}}{b_{1}} y_{2} e_{7}+g_{1} e_{1}, \\
u_{2}= & -4 \sqrt{2} e_{4} e_{6}-4 \sqrt{2} y_{6} e_{4}-4 \sqrt{2} y_{4} e_{6}+4 \sqrt{2} e_{6} e_{7} \\
& +4 \sqrt{2} y_{7} e_{6}+4 \sqrt{2} y_{6} e_{7}+3 \sqrt{2} \frac{b_{4}}{b_{3}} e_{4} e_{6} \\
& +3 \sqrt{2} \frac{b_{4}}{b_{3}} y_{6} e_{4}-3 \sqrt{2} \frac{b_{7}}{b_{3}} e_{6} e_{7}-3 \sqrt{2} \frac{b_{7}}{b_{3}} y_{6} e_{7} \\
& +4 R+g_{2} e_{3}, \\
u_{3}= & 3 e_{1} e_{4}+3 y_{4} e_{1}+3 y_{1} e_{4}-\sqrt{5} e_{1} e_{6}-\sqrt{5} y_{6} e_{1} \\
& -\sqrt{5} y_{1} e_{6}+\frac{b_{4}}{b_{5}} y_{1} e_{4}+g_{3} e_{5}, \\
& +3 \sqrt{2} \frac{b_{4}}{b_{6}} y_{3} e_{4}-3 \sqrt{2} \frac{b_{7}}{b_{6}} y_{3} e_{7}+g_{4} e_{6}, \\
u_{4}= & \sqrt{5} e_{1} e_{5}+\sqrt{5} y_{5} e_{1}+\sqrt{5} y_{1} e_{5}+\sqrt{2} e_{3} e_{4}+\sqrt{2} y_{4} e_{3} \\
& \sqrt{2} y_{3} e_{4}-\sqrt{2} e_{3} e_{7}-\sqrt{2} y_{7} e_{3}-\sqrt{2} y_{3} e_{7} \\
&
\end{aligned}
$$

when applied to the error model (32) guarantees the convergence of the errors $e_{i}(i=1, \ldots, 7)$ to zero as $t$ tends to infinity. Therefore, the states of system (30) asymptotically converge to the states of system (29) as t tends to infinity.

Proof. Consider the Lyapunov function candidate $V$ such that

$$
\begin{aligned}
V= & \frac{1}{2} b_{1} e_{1}^{2}+\frac{1}{2} b_{2} e_{2}^{2}+\frac{1}{2} b_{3} e_{3}^{2}+\frac{1}{2} b_{4} e_{4}^{2}+\frac{1}{2} b_{5} e_{5}^{2}+\frac{1}{2} b_{6} e_{6}^{2} \\
& +\frac{1}{2} b_{7} e_{7}^{2} .
\end{aligned}
$$

Using the model of the errors system given in (32), the control law given by (34), and the constraints on the control parameters $b_{2}$ and $b_{7}$ given by (33), the derivative of $V$ with respect to time is such that

$$
\begin{aligned}
\dot{V} & =b_{1} e_{1} \dot{e}_{1}+b_{2} e_{2} \dot{e_{2}}+b_{3} e_{3} \dot{e}_{3}+b_{4} e_{4} \dot{e}_{4}+b_{5} e_{5} \dot{e}_{5} \\
& +b_{6} e_{6} \dot{e}_{6}+b_{7} e_{7} \dot{e}_{7}=b_{1} e_{1}\left[-2 e_{1}-4 e_{4} e_{5}-4 y_{5} e_{4}\right. \\
& \left.-4 y_{4} e_{5}+4 e_{2} e_{7}+4 y_{7} e_{2}+4 y_{2} e_{7}-u_{1}\right] \\
& +b_{2} e_{2}\left[-9 e_{2}+3 e_{1} e_{7}+3 y_{7} e_{1}+3 y_{1} e_{7}\right] \\
& +b_{3} e_{3}\left[-4 e_{3}-4 \sqrt{2} e_{4} e_{6}-4 \sqrt{2} y_{6} e_{4}-4 \sqrt{2} y_{4} e_{6}\right. \\
& \left.+4 \sqrt{2} e_{6} e_{7}+4 \sqrt{2} y_{7} e_{6}+4 \sqrt{2} y_{6} e_{7}+4 R-u_{2}\right] \\
& +b_{4} e_{4}\left[-5 e_{4}+e_{1} e_{5}+y_{5} e_{1}+y_{1} e_{5}+3 \sqrt{2} e_{3} e_{6}\right.
\end{aligned}
$$

$$
\begin{aligned}
& \left.+3 \sqrt{2} y_{6} e_{3}+3 \sqrt{2} y_{3} e_{6}\right]+b_{5} e_{5}\left[-e_{5}+3 e_{1} e_{4}\right. \\
& +3 y_{4} e_{1}+3 y_{1} e_{4}-\sqrt{5} e_{1} e_{6}-\sqrt{5} y_{6} e_{1}-\sqrt{5} y_{1} e_{6} \\
& \left.-u_{3}\right]+b_{6} e_{6}\left[-e_{6}+\sqrt{5} e_{1} e_{5}+\sqrt{5} y_{5} e_{1}+\sqrt{5} y_{1} e_{5}\right. \\
& +\sqrt{2} e_{3} e_{4}+\sqrt{2} y_{4} e_{3}+\sqrt{2} y_{3} e_{4}-\sqrt{2} e_{3} e_{7}-\sqrt{2} y_{7} e_{3} \\
& \left.-\sqrt{2} y_{3} e_{7}-u_{4}\right]+b_{7} e_{7}\left[-5 e_{7}-7 e_{1} e_{2}-7 y_{2} e_{1}\right. \\
& \left.-7 y_{1} e_{2}-3 \sqrt{2} e_{3} e_{6}-3 \sqrt{2} y_{6} e_{3}-3 \sqrt{2} y_{3} e_{6}\right] .
\end{aligned}
$$

or

$$
\begin{aligned}
& \dot{V}=-2 b_{1} e_{1}^{2}-9 b_{2} e_{2}^{2}-4 b_{3} e_{3}^{2}-5 b_{4} e_{4}^{2}-b_{5} e_{5}^{2}-b_{6} e_{6}^{2} \\
& -5 b_{7} e_{7}^{2}+b_{1} e_{1}\left[-4 e_{4} e_{5}-4 y_{5} e_{4}-4 y_{4} e_{5}+4 e_{2} e_{7}\right. \\
& +4 y_{7} e_{2}+4 y_{2} e_{7}-u_{1}+3 \frac{b_{2}}{b_{1}} e_{2} e_{7}+3 \frac{b_{2}}{b_{1}} y_{7} e_{2} \\
& \left.+\frac{b_{4}}{b_{1}} e_{4} e_{5}+\frac{b_{4}}{b_{1}} y_{5} e_{4}-7 \frac{b_{7}}{b_{1}} e_{2} e_{7}-7 \frac{b_{7}}{b_{1}} y_{2} e_{7}\right] \\
& +b_{3} e_{3}\left[-4 \sqrt{2} e_{4} e_{6}-4 \sqrt{2} y_{6} e_{4}-4 \sqrt{2} y_{4} e_{6}\right. \\
& +4 \sqrt{2} e_{6} e_{7}+4 \sqrt{2} y_{7} e_{6}+4 \sqrt{2} y_{6} e_{7}+4 R-u_{2} \\
& +3 \sqrt{2} \frac{b_{4}}{b_{3}} e_{4} e_{6}+3 \sqrt{2} \frac{b_{4}}{b_{3}} y_{6} e_{4}-3 \sqrt{2} \frac{b_{7}}{b_{3}} e_{6} e_{7} \\
& \left.-3 \sqrt{2} \frac{b_{7}}{b_{3}} y_{6} e_{7}\right]+b_{5} e_{5}\left[3 e_{1} e_{4}+3 y_{4} e_{1}+3 y_{1} e_{4}\right. \\
& \left.-\sqrt{5} e_{1} e_{6}-\sqrt{5} y_{6} e_{1}-\sqrt{5} y_{1} e_{6}-u_{3}+\frac{b_{4}}{b_{5}} y_{1} e_{4}\right] \\
& +b_{6} e_{6}\left[\sqrt{5} e_{1} e_{5}+\sqrt{5} y_{5} e_{1}+\sqrt{5} y_{1} e_{5}+\sqrt{2} e_{3} e_{4}\right. \\
& +\sqrt{2} y_{4} e_{3}+\sqrt{2} y_{3} e_{4}-\sqrt{2} e_{3} e_{7}-\sqrt{2} y_{7} e_{3}-\sqrt{2} y_{3} e_{7} \\
& \left.-u_{4}+3 \sqrt{2} \frac{b_{4}}{b_{6}} y_{3} e_{4}-3 \sqrt{2} \frac{b_{7}}{b_{6}} y_{3} e_{7}\right]+\left[3 b_{2}-7 b_{7}\right] \\
& \text { - } y_{1} e_{2} e_{7}=-2 b_{1} e_{1}^{2}-9 b_{2} e_{2}^{2}-4 b_{3} e_{3}^{2}-5 b_{4} e_{4}^{2}-b_{5} e_{5}^{2} \\
& -b_{6} e_{6}^{2}-5 b_{7} e_{7}^{2}-g_{1} b_{1} e_{1}^{2}-g_{2} b_{3} e_{3}^{2}-g_{3} b_{5} e_{5}^{2} \\
& -g_{4} b_{6} e_{6}^{2} \text {. }
\end{aligned}
$$

Since the design parameters $b_{i}(i=1, \ldots, 7)$ and $g_{i}(i=$ $1, \ldots, 4)$ are positive constants, then it is concluded that the Lyapunov function $V$ defined by (35) is positive definite and its derivative $\dot{V}$ is negative definite. Therefore, the error functions in (31) asymptotically converge to zero as $t$ tends to infinity. Thus, the states of system (30) asymptotically converge to the states of system (29) as $t$ tends to infinity. 


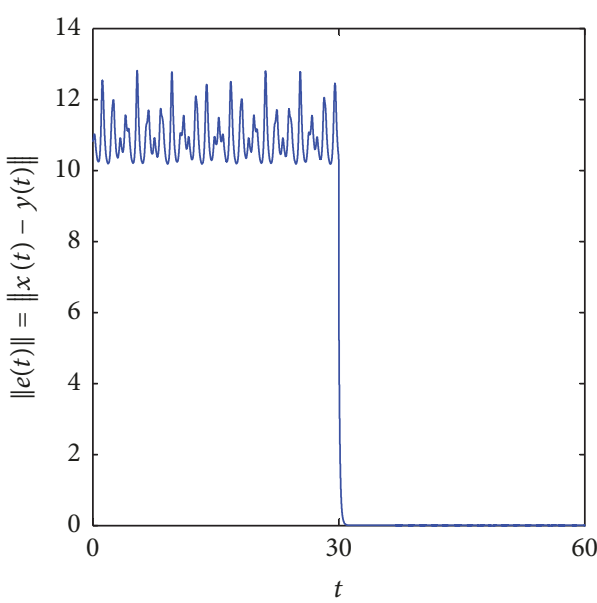

(a)

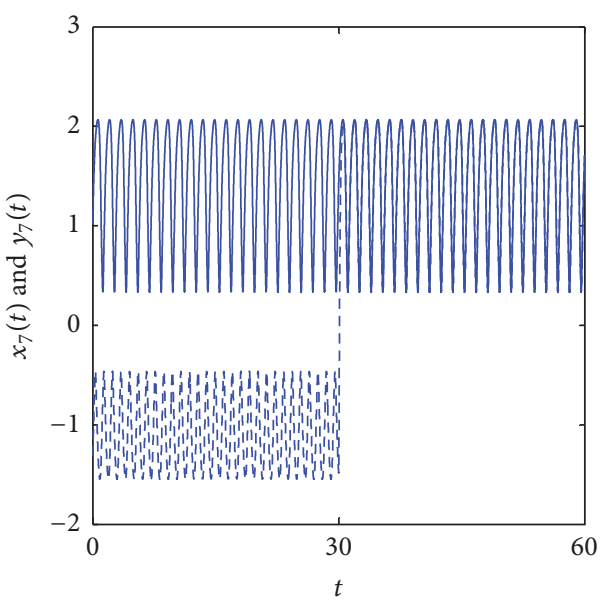

(c)

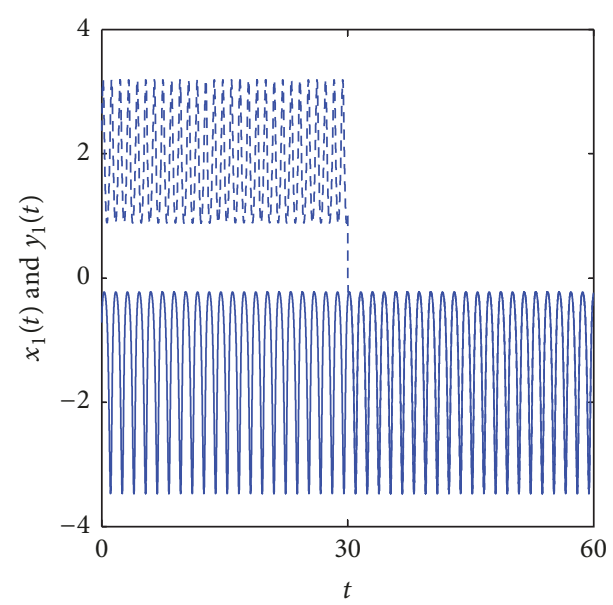

(b)

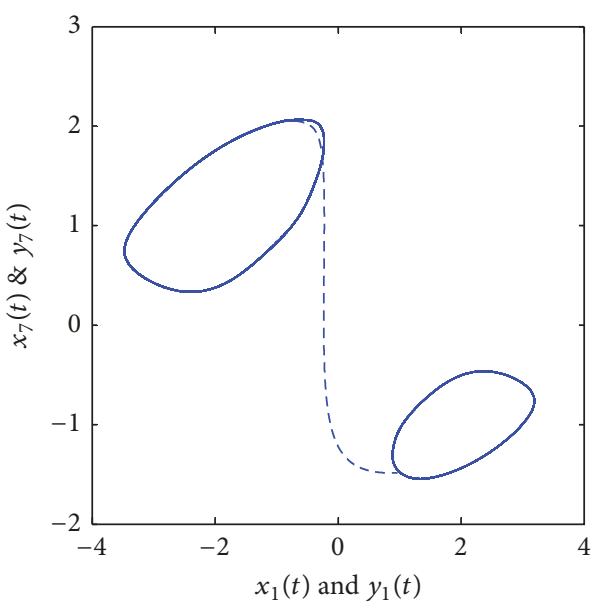

(d)

FiguRE 6: Control of a periodic orbit to a different periodic orbit when $R_{e}=26$ in system (29) and $R_{e}=23$ in system (30); the control is switched on at $t=30$. (a) The $L_{2}$ norm of the error $e$ versus time. (b) The states $x_{1}(t)$ and $y_{1}(t)$ versus time. (c) The states $x_{7}(t)$ and $y_{7}(t)$ versus time. (d) The states $x_{7}(t)$ and $y_{7}(t)$ versus $x_{1}(t)$ and $y_{1}(t)$ showing how the controller drives the states from one attractor to another attractor.

The performance of the controller in (34) when applied to the ODE system in (18) is simulated. The control gains $g_{1}, g_{2}, g_{3}$, and $g_{4}$ are taken to be 50 . Three cases are simulated. For the first case, we choose $R_{e}=$ 26 , and the initial condition $x(0)=[-0.9171-0.3868$ $0.6297-2.4110-0.0780-4.12501 .4072]^{T}$ for the system in (29) and $R_{e}=23$ and the initial condition $y(0)=[3.1934$ $-1.11941 .51193 .2126-0.66013 .9139-1.0963]^{T}$ for the system in (30). Note that these two states correspond to two stable periodic orbits with different periods. For the second case, we choose $R_{e}=23$, and $x(0)=[-0.8861$

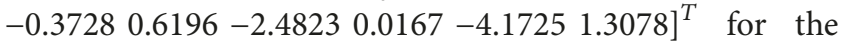
system in (29), and $R_{e}=26.377$ and $y(0)=[-0.02180 .0178$ $0.63902 .2250-0.01084 .1041-2.2075]^{T}$ for the system in (30). The first state in the second case corresponds to a periodic orbit, and the second state corresponds to a chaotic attractor or a strange attractor connecting a pair of two stable periodic orbits. For the third case, we choose $R_{e}=26.377$ and $x(0)=\left[\begin{array}{llllll}-0.0634 & -0.0596 & 0.6172 & -2.2433 & 0.0501 & -4.1940\end{array}\right.$ $2.0967]^{T}$ for the system in (29), and $R_{e}=26.377$ and $y(0)=$ $\left[\begin{array}{lllllll}0.0219 & -0.0266 & 0.6305 & 2.2267 & 0.0318 & 4.1382 & -2.1713\end{array}\right]^{T}$ for the system in (30). Note that, in this case, the two states correspond to two different chaotic or strange attractors each connecting a pair of two stable periodic orbits. Moreover, at the beginning of simulations in each case, the controllers $u_{1}, u_{2}, u_{3}$, and $u_{4}$ are set to zero for the first 30 seconds. Then the control law presented in (34) is switched on to force system (30) to synchronize with system (29). The simulation results are presented in Figure 6 for case 1. Figure 6(a) depicts the $L_{2}$ norm of the error $\|e\|=\sqrt{\sum_{i=1}^{7}\left\|x_{i}-y_{i}\right\|^{2}}$ versus time. Also, the states $x_{1}(t)$ and $y_{1}(t)$ versus time and $x_{7}(t)$ and $y_{7}(t)$ versus time are plotted in Figures 6(b) and 6(c), 


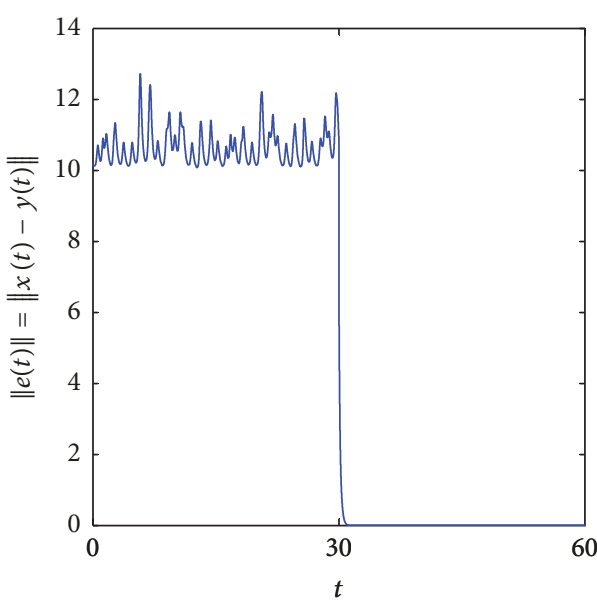

(a)

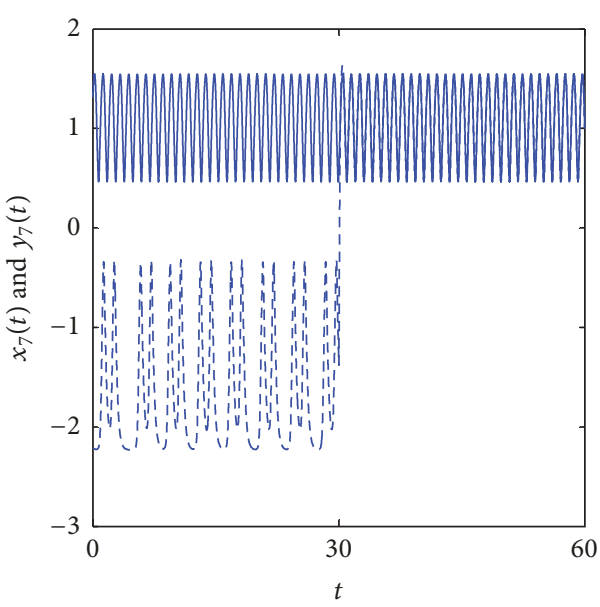

(c)

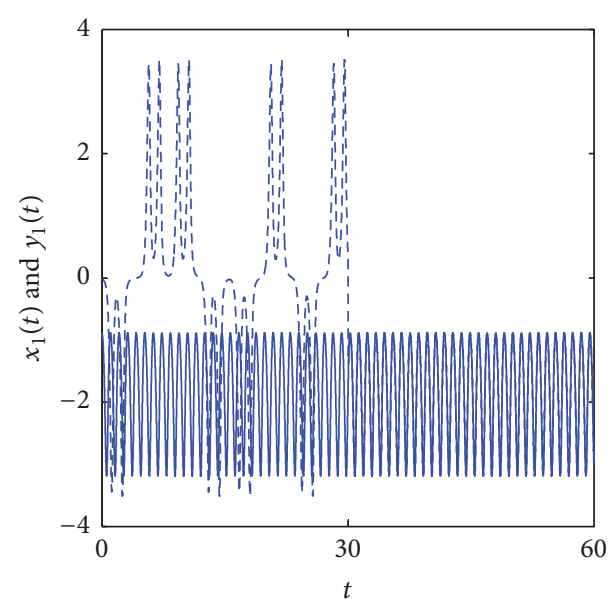

(b)

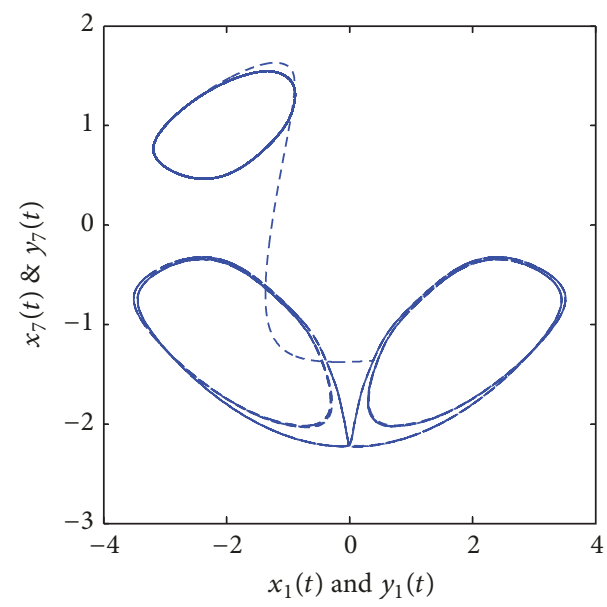

(d)

FIGURE 7: Control of a chaotic attractor to a periodic orbit when $R_{e}=23$ in system (29) and $R_{e}=26.377$ in system (30); the control is switched on at $t=30$. (a) The $L_{2}$ norm of the error $e$ versus time. (b) The states $x_{1}(t)$ and $y_{1}(t)$ versus time. (c) The states $x_{7}(t)$ and $y_{7}(t)$ versus time. (d) The states $x_{7}(t)$ and $y_{7}(t)$ versus $x_{1}(t)$ and $y_{1}(t)$ showing how the controller drives the states from one chaotic attractor to a periodic orbit.

respectively. Figure $6(\mathrm{~d})$ plots the states $x_{7}(t)$ and $y_{7}(t)$ versus $x_{1}(t)$ and $y_{1}(t)$; the figure shows the effectiveness of the controller to drive the dynamics from one attractor to a different attractor. Figure 7 shows the simulation results for case 2, and Figure 8 shows the results for case 3 . In the three cases, it is shown how the error converges to zero. Hence, it can be concluded that the designed control law in (34) is able to synchronize the ODE systems in (29) and (30) when these systems have the same or different Reynolds numbers but they start from two different initial conditions.

\section{Conclusion}

This paper investigates the symmetries, dynamics, and control of the 2D Kolmogorov flow with a forcing in the mode $(0,2)$. First, a seventh-order system of ODEs is constructed using the Fourier series expansion. A computer analysis shows that the constructed system exhibits a chaotic behavior for a certain range of the Reynolds number. In addition, the ODE system shows a homoclinic gluing bifurcation similar to the original PDE simulation. Then, Lyapunov based controllers are designed to control the dynamics of ODEs to a fixed point, a periodic orbit, or a chaotic or strange attractor. Extensive numerical studies are presented to show the effectiveness of the proposed controllers.

Future work will address the design of adaptive control schemes for the reduced order ODE model of the 2D Kolmogorov flow.

\section{Conflicts of Interest}

The author declares that there are no conflicts of interest regarding the publication of this paper. 


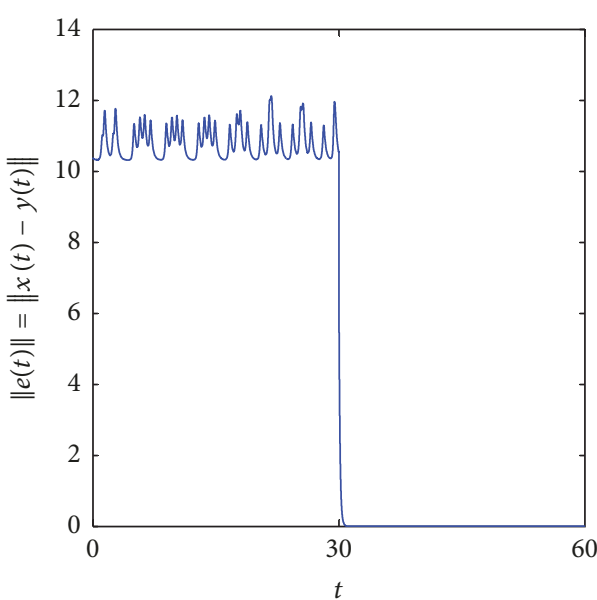

(a)

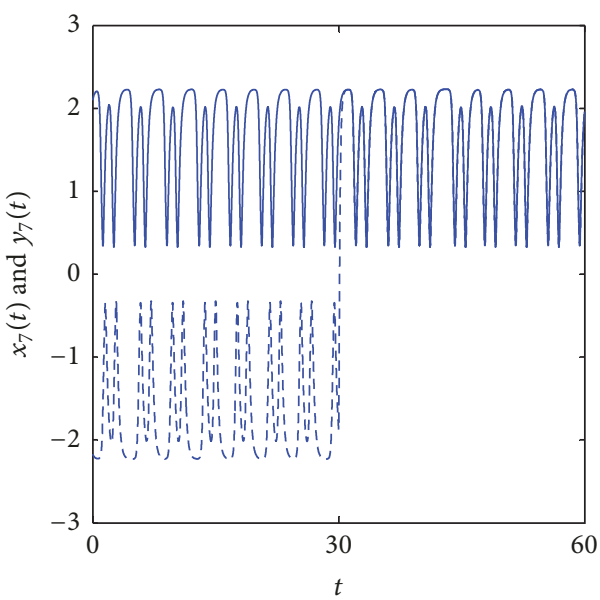

(c)

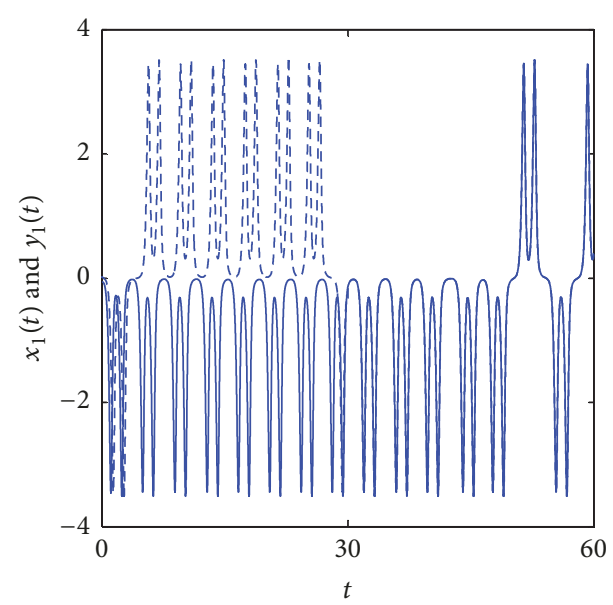

(b)

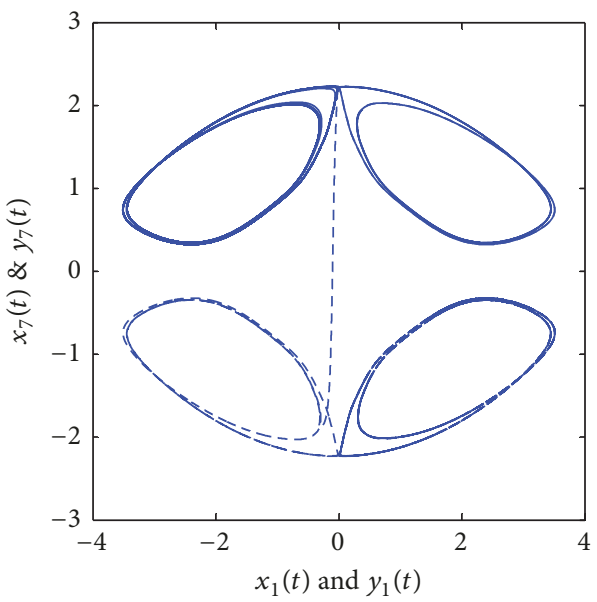

(d)

FIGURE 8: Control of a chaotic attractor to a different chaotic attractor when $R_{e}=26.377$ in system (29) and $R_{e}=26.377$ in system (30); the control is switched on at $t=30$. (a) The $L_{2}$ norm of the error $e$ versus time. (b) The states $x_{1}(t)$ and $y_{1}(t)$ versus time. (c) The states $x_{7}(t)$ and $y_{7}(t)$ versus time. (d) The states $x_{7}(t)$ and $y_{7}(t)$ versus $x_{1}(t)$ and $y_{1}(t)$ showing how the controller drives the states from one chaotic attractor to a different chaotic attractor.

\section{Acknowledgments}

This research was supported and funded by the Research Sector, Kuwait University, under Grant no. SM07/16.

\section{References}

[1] E. S. Titi, "On approximate inertial manifolds to the NavierStokes equations," Journal of Mathematical Analysis and Applications, vol. 149, no. 2, pp. 540-557, 1990.

[2] P. D. Christofides and P. Daoutidis, "Finite-dimensional control of parabolic PDE systems using approximate inertial manifolds," Journal of Mathematical Analysis and Applications, vol. 216, no. 2, pp. 398-420, 1997.

[3] A. Debussche and M. Marion, "On the construction of families of approximate inertial manifolds," Journal of Differential Equations, vol. 100, no. 1, pp. 173-201, 1992.

[4] E. Fabes, M. Luskin, and G. R. Sell, "Construction of inertial manifolds by elliptic regularization," Journal of Differential Equations, vol. 89, no. 2, pp. 355-387, 1991.
[5] C. Foias and R. Temam, "The algebraic approximation of attractors: the finite-dimensional case," Physica D: Nonlinear Phenomena, vol. 32, no. 2, pp. 163-182, 1988.

[6] R. Temam, "Induced trajectories and approximate inertial manifolds," Mathematical Modelling and Numerical Analysis, vol. 23, no. 3, pp. 541-561, 1989.

[7] J. Zhang, S. Ren, and G. Mei, "Model reduction on inertial manifolds for N-S equations approached by multilevel finite element method," Communications in Nonlinear Science and Numerical Simulation, vol. 16, no. 1, pp. 195-205, 2011.

[8] C. Foias, G. R. Sell, and R. Temam, "Inertial manifolds for nonlinear evolutionary equations," Journal of Differential Equations, vol. 73, no. 2, pp. 309-353, 1988.

[9] M. Kwak, "Finite-dimensional inertial forms for the 2D NavierStokes equations," Indiana University Mathematics Journal, vol. 41, no. 4, pp. 927-981, 1992.

[10] C. Foias, M. S. Jolly, I. G. Kevrekidis, G. . Sell, and E. S. Titi, "On the computation of inertial manifolds," Physics Letters A, vol. 131, no. 7-8, pp. 433-436, 1988. 
[11] M. Jolly, "Bifurcation computations on an approximate inertial manifold for the 2D Navier-Stokes equations," Physica D: Nonlinear Phenomena, vol. 63, no. 1-2, pp. 8-20, 1993.

[12] C. Boldrighini and V. Franceschini, "A five-dimensional truncation of the plane incompressible Navier-Stokes equations," Communications in Mathematical Physics, vol. 64, no. 2, pp. 159$170,1978 / 79$.

[13] D. Baive and V. Franceschini, "Symmetry breaking on a model of five-mode truncated Navier-Stokes equations," Journal of Statistical Physics, vol. 26, no. 3, pp. 471-484, 1981.

[14] V. Franceschini, G. Inglese, and C. Tebaldi, "A five-mode truncation of the Navier-Stokes equations on a three-dimensional torus," Computational Mechanics, vol. 3, no. 1, pp. 19-37, 1988.

[15] V. Franceschini, C. Giberti, and M. Nicolini, "Common periodic behavior in larger and larger truncations of the Navier-Stokes equations," Journal of Statistical Physics, vol. 50, no. 5-6, pp. 879896, 1988.

[16] V. Franceschini and C. Tebaldi, "Sequences of infinite bifurcations and turbulence in a five-mode truncation of the NavierStokes equations," Journal of Statistical Physics, vol. 21, no. 6, pp. 707-726, 1979.

[17] V. Franceschini and C. Tebaldi, "A seven-mode truncation of the plane incompressible Navier-Stokes equations," Journal of Statistical Physics, vol. 25, no. 3, pp. 397-417, 1981.

[18] Z.-M. Chen, "Bifurcations of a steady-state solution to the two-dimensional Navier-Stokes equations," Communications in Mathematical Physics, vol. 201, no. 1, pp. 117-138, 1999.

[19] Z. Chen and W. Price, "Transition to chaos in fluid motion system," Chaos, Solitons \& Fractals, vol. 26, no. 4, pp. 1195-1202, 2005.

[20] Z.-M. Chen and W. G. Price, "Chaotic behavior of a Galerkin model of a two-dimensional flow," Chaos: An Interdisciplinary Journal of Nonlinear Science, vol. 14, no. 4, pp. 1056-1068, 2004.

[21] V. X. Liu, "Instability for the Navier-Stokes equations on the 2-dimensional torus and a lower bound for the Hausdorff dimension of their global attractors," Communications in Mathematical Physics, vol. 147, no. 2, pp. 217-230, 1992.

[22] A. Thess, "Instabilities in two-dimensional spatially periodic flows. I. Kolmogorov flow," Physics of Fluids A. Fluid Dynamics, vol. 4, no. 7, pp. 1385-1395, 1992.

[23] B. Nicolaenko and Z.-S. She, "Symmetry-breaking homoclinic chaos and vorticity bursts in periodic Navier-Stokes flows," European Journal of Mechanics - B/Fluids, vol. 10, no. 2, suppl., pp. 67-74, 1991.

[24] L. Sirovich, "Turbulence and the dynamics of coherent structures," Quarterly of Applied Mathematics, vol. 45, no. 3, pp. 561590, 1987.

[25] D. Armbruster, B. Nicolaenko, N. Smaoui, and P. Chossat, "Symmetries and dynamics for 2-D Navier-Stokes flow," Physica D: Nonlinear Phenomena, vol. 95, no. 1, pp. 81-93, 1996.

[26] D. Armbruster, R. Heiland, E. J. Kostelich, and B. Nicolaenko, "Phase-space analysis of bursting behavior in Kolmogorov flow," Physica D: Nonlinear Phenomena, vol. 58, no. 1-4, pp. 392-401, 1992.

[27] N. Smaoui and D. Armbruster, "Symmetry and the KarhunenLoeve analysis," SIAM Journal on Scientific Computing, vol. 18, no. 5, pp. 1526-1532, 1997.

[28] N. Smaoui, "A model for the unstable manifold of the bursting behavior in the 2D Navier-Stokes flow," SIAM Journal on Scientific Computing, vol. 23, no. 3, pp. 824-839, 2001.
[29] N. Smaoui, "Linear versus nonlinear dimensionality reduction of high-dimensional dynamical systems," SIAM Journal on Scientific Computing, vol. 25, no. 6, pp. 2107-2125, 2004.

[30] F. Abergel and R. Temam, "On some control problems in fluid mechanics," Theoretical and Computational Fluid Dynamics, vol. 1, no. 6, pp. 303-325, 1990.

[31] J. Baker and P. D. Christofides, "Finite-dimensional approximation and control of non-linear parabolic PDE systems," International Journal of Control, vol. 73, no. 5, pp. 439-456, 2000.

[32] J. Baker, A. Armaou, and P. . Christofides, "Nonlinear control of incompressible fluid flow: application to Burgers' equation and 2D channel flow," Journal of Mathematical Analysis and Applications, vol. 252, no. 1, pp. 230-255, 2000.

[33] A. Armaou and P. D. Christofides, "Wave suppression by nonlinear finite-dimensional control," Chemical Engineering Science, vol. 55, no. 14, pp. 2627-2640, 2000.

[34] S. Guan, Y. C. Zhou, G. W. Wei, and C.-H. Lai, "Controlling flow turbulence," Chaos: An Interdisciplinary Journal of Nonlinear Science, vol. 13, no. 1, pp. 64-70, 2003.

[35] G. Gambino, M. C. Lombardo, and M. Sammartino, "Adaptive control of a seven mode truncation of the Kolmogorov flow with drag," Chaos, Solitons \& Fractals, vol. 41, no. 1, pp. 47-59, 2009.

[36] N. Smaoui and M. Zribi, "Dynamics and control of the 2-d Navier-Stokes equations," Applied Mathematics and Computation, vol. 237, pp. 461-473, 2014.

[37] N. Smaoui and M. Zribi, "Dynamics and control of the sevenmode truncation system of the 2-d Navier-Stokes equations," Communications in Nonlinear Science and Numerical Simulation, vol. 32, pp. 169-189, 2016.

[38] N. Smaoui and M. Zribi, "On the control of the chaotic attractors of the 2-d Navier-Stokes equations," Chaos: An Interdisciplinary Journal of Nonlinear Science, vol. 27, no. 3, Article ID 033111, 033111, 22 pages, 2017.

[39] V. Arnold and L. D. Mechalkin, "The Kolmogorov seminar on analysis," Russian Mathematical Surveys, vol. 15, pp. 247-250, 1958.

[40] J. Guckenheimer, M. R. Myers, F. J. Wicklin, and P. A. Worfolk, dstool: A dynamical system toolkit with an interactive graphical interface, User's Manual, Center for Applied Mathematics, Cornell University, 1995.

[41] O. A. Ladyzhenskaya, The Mathematical Theory of Viscous Incompressible Flow, Gordon and Breach, New York, NY, USA, 1965. 


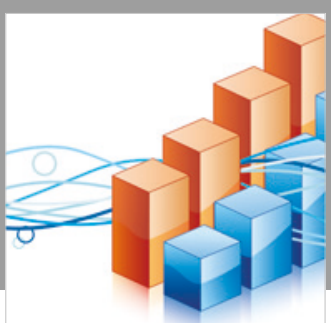

Advances in

Operations Research

\section{-n-m}
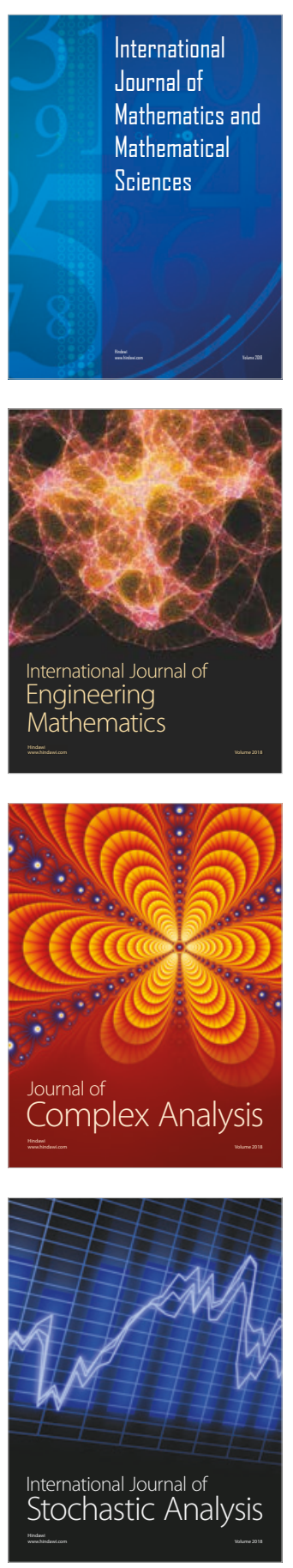
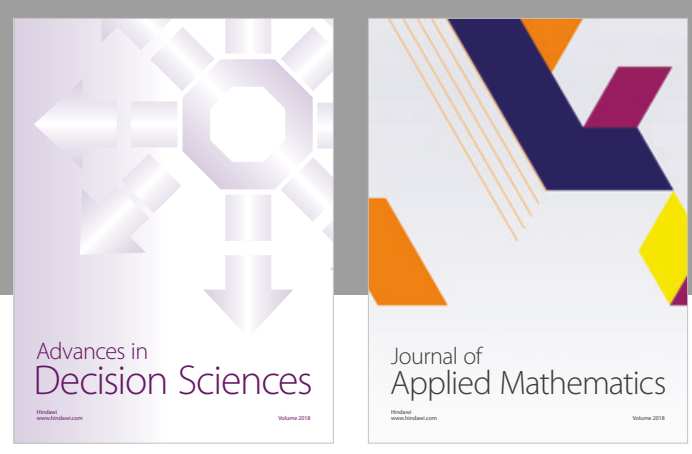

Journal of

Applied Mathematics
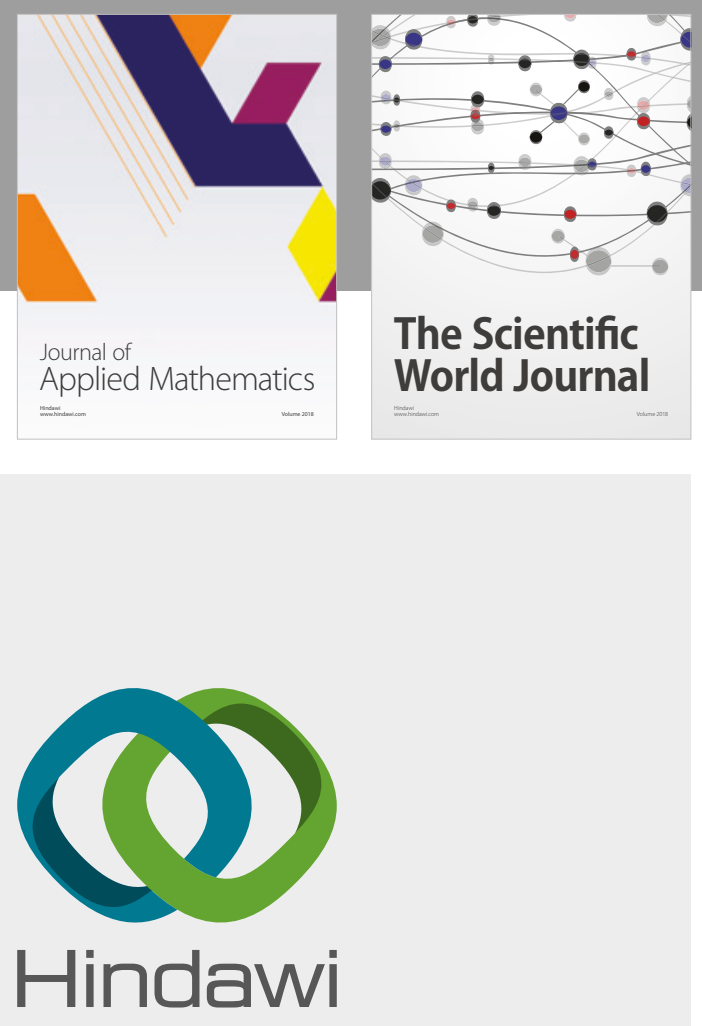

Submit your manuscripts at

www.hindawi.com

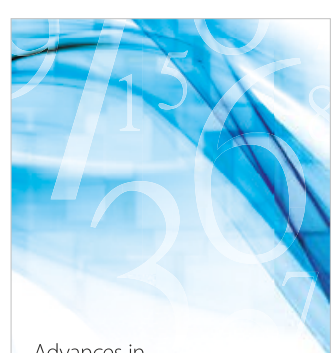

Advances in
Numerical Analysis
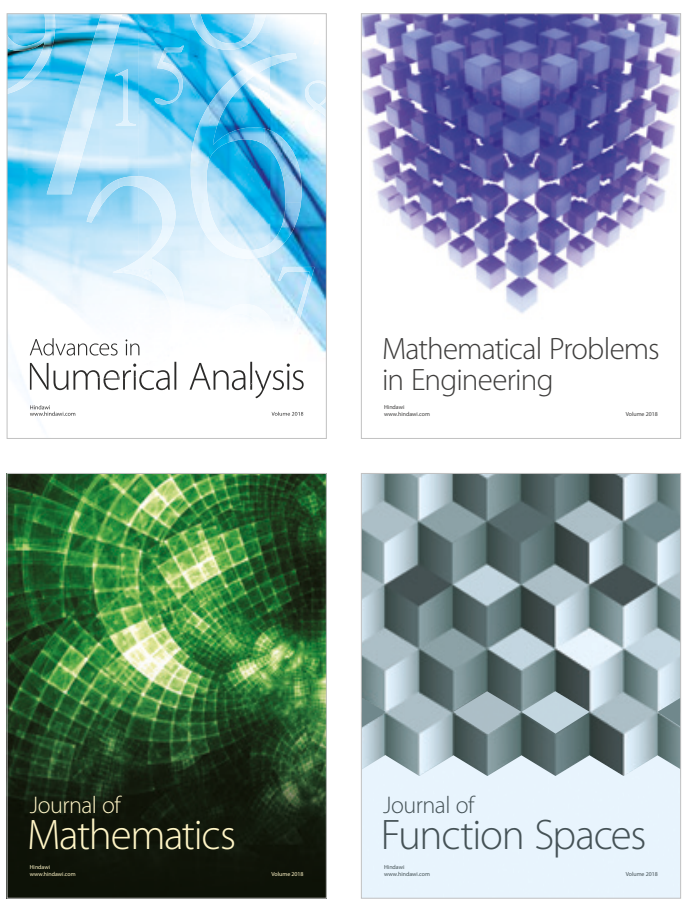

Mathematical Problems in Engineering

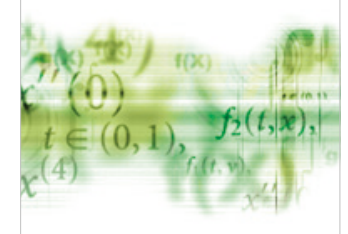

International Journal of

Differential Equations

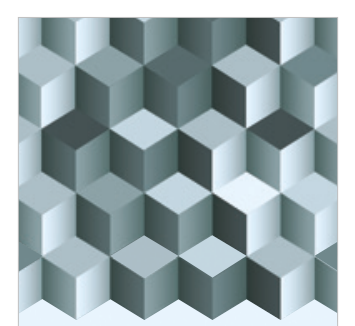

Journal of

Function Spaces
The Scientific

World Journal

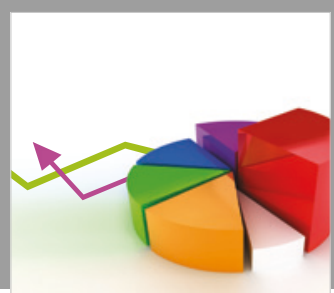

Journal of

Probability and Statistics
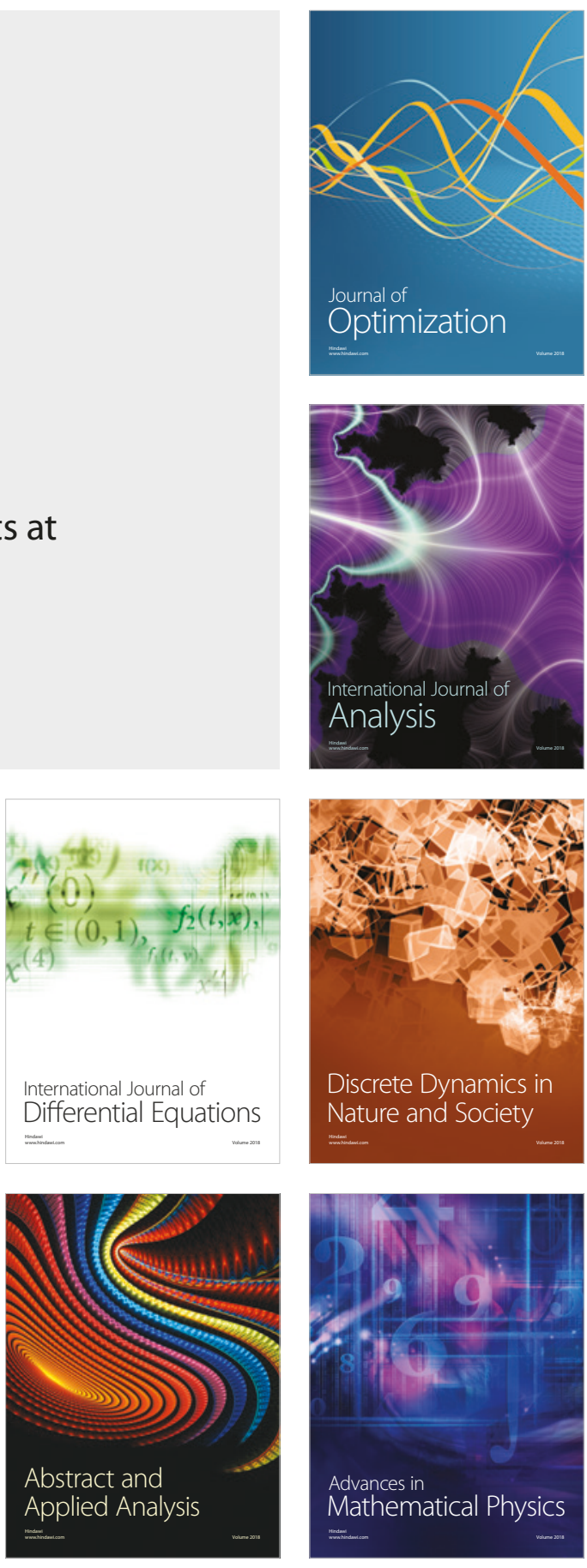